\title{
Diagnosis and management of glycogen storage disease type I: a practice guideline of the American College of Medical Genetics and Genomics
}

\author{
Priya S. Kishnani, MD¹, Stephanie L. Austin, MS, MA¹, Jose E. Abdenur, MD², Pamela Arn, MD², \\ Deeksha S. Bali, PhD1', Anne Boney, MED, RD1, Wendy K. Chung, MD, PhD ${ }^{4}$, Aditi I. Dagli, MD ${ }^{5}$,

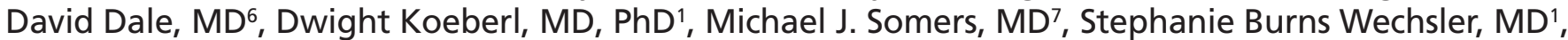 \\ David A. Weinstein, MD, MMSc ${ }^{5}$, Joseph I. Wolfsdorf, MB, BCh ${ }^{7}$ and Michael S. Watson, MS, PhD ${ }^{8}$
}

\begin{abstract}
Disclaimer: This guideline is designed primarily as an educational resource for clinicians to help them provide quality medical services. Adherence to this guideline is completely voluntary and does not necessarily ensure a successful medical outcome. This guideline should not be considered inclusive of all proper procedures and tests or exclusive of other procedures and tests that are reasonably directed toward obtaining the same results. In determining the propriety of any specific procedure or test, the clinician should apply his or her own professional judgment to the specific clinical circumstances presented by the individual patient or specimen. Clinicians are encouraged to document the reasons for the use of a particular procedure or test, whether or not it is in conformance with this guideline. Clinicians also are advised to take notice of the date this guideline was adopted and to consider other medical and scientific information that becomes available after that date. It also would be prudent to consider whether intellectual property interests may restrict the performance of certain tests and other procedures.
\end{abstract}

Purpose: Glycogen storage disease type I (GSD I) is a rare disease of variable clinical severity that primarily affects the liver and kidney. It is caused by deficient activity of the glucose 6-phosphatase enzyme (GSD Ia) or a deficiency in the microsomal transport proteins for glucose 6-phosphate (GSD Ib), resulting in excessive accumulation of glycogen and fat in the liver, kidney, and intestinal mucosa. Patients with GSD I have a wide spectrum of clinical manifestations, including hepatomegaly, hypoglycemia, lactic acidemia, hyperlipidemia, hyperuricemia, and growth retardation. Individuals with GSD type Ia typically have symptoms related to hypoglycemia in infancy when the interval between feedings is extended to 3-4 hours. Other manifestations of the disease vary in age of onset, rate of disease progression, and severity. In addition, patients with type Ib have neutropenia, impaired neutrophil function, and inflammatory bowel disease. This guideline for the management of GSD I was developed as an educational resource for health-care providers to facilitate prompt, accurate diagnosis and appropriate management of patients.

Methods: A national group of experts in various aspects of GSD I met to review the evidence base from the scientific literature and provided their expert opinions. Consensus was developed in each area of diagnosis, treatment, and management.
Results: This management guideline specifically addresses evaluation and diagnosis across multiple organ systems (hepatic, kidney, gastrointestinal/nutrition, hematologic, cardiovascular, reproductive) involved in GSD I. Conditions to consider in the differential diagnosis stemming from presenting features and diagnostic algorithms are discussed. Aspects of diagnostic evaluation and nutritional and medical management, including care coordination, genetic counseling, hepatic and renal transplantation, and prenatal diagnosis, are also addressed.

Conclusion: A guideline that facilitates accurate diagnosis and optimal management of patients with GSD I was developed. This guideline helps health-care providers recognize patients with all forms of GSD I, expedite diagnosis, and minimize adverse sequelae from delayed diagnosis and inappropriate management. It also helps to identify gaps in scientific knowledge that exist today and suggests future studies.

Genet Med advance online publication 6 November 2014

Key Words: glycogen storage disease; glycogen storage disease type I; von Gierke disease

\section{PURPOSE}

This guideline is intended as an educational resource. It highlights current practices and therapeutic approaches to the diagnosis and management of GSD I and its early and longterm complications.

\section{GENERAL BACKGROUND}

\section{History}

In 1929, von Gierke described glycogen storage disease type I (GSD I) after reviewing the autopsy reports of two children whose livers and kidneys contained excessive amounts of

\footnotetext{
${ }^{1}$ Department of Pediatrics, Duke University Medical Center, Durham, North Carolina, USA; ${ }^{2}$ Division of Metabolic Disorders, Children's Hospital of Orange County, Orange, California, USA; ${ }^{3}$ Division of Genetics, Nemours Children's Clinic, Jacksonville, Florida, USA; ${ }^{4}$ Departments of Pediatrics and Medicine, Columbia University Medical Center, New York, New York, USA; ${ }^{5}$ Department of Pediatrics, University of Florida College of Medicine, Gainesville, Florida, USA; ${ }^{6}$ Department of Medicine, University of Washington, Seattle, Washington, USA; ${ }^{7}$ Division of Endocrinology, Boston Children's Hospital, Boston, Massachusetts, USA; ${ }^{8}$ American College of Medical Genetics and Genomics, Bethesda, Maryland, USA. Correspondence: Michael S. Watson (mwatson@acmg.net)
} 
glycogen. ${ }^{1}$ In 1952, Cori and Cori ${ }^{2}$ reported six similar patients and discovered that the absence of the enzyme glucose-6-phosphatase (G6Pase) caused von Gierke disease, establishing the first metabolic disorder in which an enzyme defect was identified. Two patients had almost total deficiency of hepatic G6Pase; the remaining four patients had normal enzyme activity. Early on, the authors recognized the variability of the hepatic GSDs. In 1978, Narisawa et al. ${ }^{3}$ explained the paradox of the four patients with GSD and normal enzyme activity when he described GSD type Ib (GSD Ib) and showed that it was caused by deficiency of the transporter enzyme glucose-6-phosphate translocase (G6PT). Deficiency of the enzyme G6Pase results in GSD Ia, and deficiency of G6PT results in GSD Ib.

The human G6Pase gene, G6PC, is a single-copy gene (OMIM\# 613742) located on chromosome 17q21, which was cloned in 1993 by Lei et al. ${ }^{4}$ The group identified mutations causing GSD type Ia (GSD Ia) and generated a G6Pase-deficient mouse model in $1996 .{ }^{5}$ G6PC spans $\sim 12.5 \mathrm{~kb}$ and consists of five coding exons. The human G6PT gene, SLC37A4 (OMIM\# 602671), which causes GSD Ib, was cloned and found to be located on chromosome 11q23. SLC37A4 spans $\sim 5.3 \mathrm{~kb}$ and contains nine exons. Approximately $80 \%$ of people with GSD I have type Ia and $20 \%$ have type Ib.

\section{Overview and general background}

G6Pase is a multipart enzyme system located in the endoplasmic reticulum membrane. G6Pase together with the glucose6-phosphate transporter (SLC37A4/G6PT) forms the complex responsible for glucose production by catalyzing the terminal step of both glycogenolysis and gluconeogenesis. It is a key enzyme in regulation of blood glucose (BG) levels. Deficiency of glucose 6-phosphatase activity or its microsomal transport proteins results in excessive accumulation of glycogen and fat in the liver, kidney, and intestinal mucosa.

The presenting symptoms of GSD Ia vary according to the patient's age. Patients with GSD I may present during the neonatal period with hypoglycemia and lactic acidosis; however, they more commonly present at 3 to 6 months of age with hepatomegaly and/or signs and symptoms of hypoglycemia, including seizures. Clinical characteristics include doll-like facies, poor growth, short stature, and a distended abdomen due to pronounced hepatomegaly and nephromegaly. Biochemical manifestations include hypoglycemia, hyperlipidemia, hypertriglyceridemia, hyperlactatemia, and hyperuricemia. Patients with type Ib also have neutropenia and impaired neutrophil function, resulting in recurrent bacterial infections and oral and intestinal mucosa ulceration. Neutropenia may also be observed in a subset of GSD Ia patients. ${ }^{6}$ Patients with GSD I do not have skeletal myopathy or increased creatine kinase levels, which are characteristic of GSD type IIIa.

GSD I is an autosomal recessive, pan-ethnic disorder with genetic mutations identified in Caucasians, Ashkenazi Jews, Hispanics, and Asians. ${ }^{7-15}$ The overall incidence of the disease is $\sim 1 / 100,000$. The disease prevalence is relatively high in the Ashkenazi Jewish population (prevalence 1/20,000). There are many known pathogenic mutations in both G6PC and SLC37A4 genes. However, some ethnic group-specific common mutations account for $\sim 90 \%$ of known disease alleles. ${ }^{7,12,16,17}$ Depending on the specific ethnic group, these common mutations can account for $100 \%$ of disease alleles.

\section{Clinical history}

The diagnosis is based on the clinical presentation, specific constellation of biochemical abnormalities, molecular genetic testing, and/or enzymology in liver biopsy tissue. ${ }^{18}$ Symptomatic hypoglycemia may appear soon after birth; however, most patients are asymptomatic as long as they receive frequent feedings that contain sufficient glucose to prevent hypoglycemia. Symptoms of hypoglycemia typically appear only when the interval between feedings increases, such as when the infant starts to sleep through the night or when an intercurrent illness disrupts normal patterns of feeding. Very rarely, hypoglycemia may be mild, causing a delay in the diagnosis until adulthood when liver adenomas and hyperuricemia are detected. ${ }^{19}$

Patients may present with hyperpnea due to lactic acidosis, which may simulate that occurring in pneumonia. The condition may not be recognized until the infant is several months old with an enlarged liver and protuberant abdomen noted on a routine physical examination. Ultrasound imaging of the liver is similar in GSD I, GSD III, and several other liver storage disorders. However, the presence of nephromegaly and the characteristic biochemical abnormalities seen in GSD I provide clues to the diagnosis. ${ }^{21}$

Untreated patients typically appear short for age, with a round face and full cheeks, giving them a cushingoid appearance. They have failure to thrive and delayed motor development. Cognitive development is usually normal unless the patient has cerebral damage from recurrent hypoglycemic episodes. During infancy, the BG concentration may decrease to $<40 \mathrm{mg} / \mathrm{dl}(2.2 \mathrm{mmol} / \mathrm{l})$ within 3-4 hours of a feeding. Longer intervals between feedings cause more severe hypoglycemia accompanied by lactic acidemia and metabolic acidosis.

Long-term complications are common and are beginning to be more recognized and understood. In most individuals with GSD I, hepatomegaly decreases with age; however, development of liver adenomas is common with increasing age, and some individuals develop hepatocellular carcinoma (HCC). ${ }^{22-}$ ${ }^{26}$ GSD I has been associated with a bleeding diathesis due to impaired platelet function ${ }^{27}$ and/or a von Willebrand-like platelet defect. ${ }^{28}$ Anemia is also noted, especially in patients with hepatic adenomas. ${ }^{29}$ Vitamin D deficiency is increasingly recognized in these patients. ${ }^{30-35}$ Individuals with GSD I have decreased bone mass and are at increased risk for osteoporosis and fractures. ${ }^{36}$ Proximal renal tubular dysfunction is common in inadequately treated patients, and patients with poorly controlled conditions may develop renal glomerular dysfunction that can progress to renal failure and require a renal transplant. ${ }^{37-40}$ Liver transplant (LT) or a combined liver and kidney transplant is needed in some cases. ${ }^{41-43}$ Menorrhagia appears to be a problem in females of reproductive age with GSD I. ${ }^{44,45}$ 
Polycystic ovaries have been documented in females with GSD I after 4 years of age, but fertility is not thought to be reduced. ${ }^{46-48}$ Pulmonary hypertension has also been reported in patients. ${ }^{49,50}$ The serum of untreated patients is often cloudy or milky with very high triglyceride concentrations and moderately increased levels of phospholipids, total lipoprotein cholesterol, and low-density lipoprotein cholesterol; by contrast, the concentration of high-density lipoprotein cholesterol is low. Early atherosclerosis with risk for ischemic stroke is a potential long-term concern. ${ }^{51}$ Acute pancreatitis may occur in some patients with severe hyperlipidemia, especially in individuals with severe persistent hypertriglyceridemia $(>1,000 \mathrm{mg} / \mathrm{dl}){ }^{18}$ Patients with GSD Ib, and on occasion those with Ia, are at increased risk for Crohn disease-like enterocolitis. ${ }^{52}$

The circulating concentration of free fatty acids is markedly increased, whereas blood $\beta$-hydroxybutyrate levels are only mildly or moderately increased relative to the corresponding free fatty acid levels. . $3,54^{2}$

Eruptive xanthomata may appear on the extensor surfaces of the extremities and on the buttocks. ${ }^{55}$ These findings are becoming less common with increased awareness and earlier diagnosis.

A bleeding tendency manifested as recurrent epistaxis in childhood followed later in life by easy bruising and/or oozing after dental or other surgeries, as well as menorrhagia in menstruating females is caused by impaired platelet function and/ or an acquired von Willebrand-like disease..$^{45}$ Reduced platelet adhesiveness, abnormal platelet aggregation, and impaired release of adenosine diphosphate in response to collagen and epinephrine have been observed. The platelet defects are secondary to the systemic metabolic abnormalities and may be corrected by improving control of the metabolic state. . $^{18,27,56,57}$

Although hypoglycemia becomes less severe with increasing age, inadequate therapy causes pronounced impairment of physical growth, delayed onset of puberty, and many longterm sequelae of the disease. However, normal growth can occur, provided that patients maintain good metabolic control at an early age. ${ }^{58}$

A majority of patients with GSD I have nephromegaly that is readily demonstrable by ultrasonography ${ }^{37,59}$ Proximal tubular dysfunction (glucosuria, phosphaturia, hypokalemia, and a generalized aminoaciduria) may be observed in untreated or inadequately treated patients. The proximal tubular dysfunction is reversible with improved metabolic control of the disease. ${ }^{37,60}$ Some patients have a distal renal tubular acidification defect associated with hypocitraturia and hypercalciuria, predisposing them to nephrocalcinosis and renal calculi. ${ }^{40,61}$ Increased urinary albumin excretion (microalbuminuria) due to hyperfiltration may occur in adolescents and young adults with GSD I, similar to what is seen in diabetic patients.

Severe renal injury with proteinuria, hypertension, and decreased creatinine clearance due to focal segmental glomerulosclerosis and interstitial fibrosis, ultimately leading to endstage renal disease, may also be seen in young adults. ${ }^{62}$ Patients with persistently elevated blood lactate, serum lipids, and uric acid levels appear more at risk for nephropathy..$^{58,63}$
Patients with GSD Ib have similar clinical and biochemical abnormalities in addition to neutropenia (persistent or cyclic) - the severity of which varies from mild to complete agranulocytosis-associated with recurrent bacterial infections ${ }^{64}$ Children with GSD Ib are prone to oral complications, including recurrent mucosal ulceration, gingivitis, and rapidly progressive periodontal disease. They frequently develop inflammatory bowel disease (Crohn disease-like enterocolitis) and may have an increased prevalence of thyroid autoimmunity and hypothyroidism. ${ }^{65}$

\section{METHODS/PROCESS}

\section{Consensus development panel}

A national group of experts in clinical and laboratory diagnosis, treatment and management (cardiovascular, gastrointestinal/ nutrition, hepatic, reproductive, neuromuscular), and genetic aspects of GSD I was assembled to review the evidence base and develop management guidelines. After a meeting during which published material and personal experience were reviewed by the panel, experts in the various areas reviewed the literature in these areas and drafted the guidelines. The participants provided conflict of interest statements and their conflicts are stated in the Acknowledgments section. All members of the panel reviewed and approved the final guidelines. Consensus was defined as agreement among all members of the panel. For the most part, the evidence and resulting recommendations are considered expert opinion because additional levels of evidence were not available in the literature. Penultimate drafts of these guidelines were shared with an external review group consisting of Yuan-Tsong Chen, Philippe Labrune, Areeg El-Gharbawy, and Kathy Ross. The working group considered their suggestions and changes were made as considered appropriate.

\section{Target audience}

This guideline is directed at a wide range of care providers. Although care is commonly provided by metabolic disease specialists/biochemical geneticists, gastroenterologists, and endocrinologists in conjunction with a clinical nutritionist (dietician), it is important that primary-care providers and other specialists who often are involved in the care of individuals with GSD I also be able to recognize the condition and provide appropriate care for these patients.

\section{DIAGNOSTIC CONFIRMATION}

\section{Differential diagnosis}

The diagnosis in a classic case of GSD I is usually straightforward. The principal differential diagnosis includes other forms of GSD associated with hepatomegaly and hypoglycemia, especially GSD type III and Fanconi-Bickel syndrome, a glucose transporter 2 transporter defect classified as GSD XI, which is not involved in the glycogen metabolism pathway (Table 1), and, possibly, GSD VI and IX. GSD I and III have several features in common, including hepatomegaly, hypoglycemia, and hyperlipidemia. However, some key differences between GSD I and GSD III help to differentiate these two disorders. Patients 
with GSD I typically present earlier (in the first few months of life) with severe fasting hypoglycemia within 3-4 hours after feeding. Hypoglycemia is usually not as severe in patients with GSD III because gluconeogenesis is intact and the peripheral branches of the glycogen molecule can be mobilized by the action of hepatic phosphorylase. Nonetheless, for reasons that are not well understood, some patients with GSD III have an early clinical onset and experience severe hypoglycemia after a brief period without feeding. ${ }^{66}$

Blood lactate levels increase rapidly in GSD I as BG concentrations decrease to levels that normally trigger a counterregulatory response $(<70 \mathrm{mg} / \mathrm{dl}$ or $4 \mathrm{mmol} / \mathrm{l})$ and are markedly increased when BG levels decrease to $<40-50 \mathrm{mg} / \mathrm{dl}$ or $2.2-2.8$ $\mathrm{mmol} / \mathrm{l})$. Blood $\beta$-hydroxybutyrate levels increase only modestly in GSD I ${ }^{53,54}$ in contrast to marked hyperketonemia with fasting hypoglycemia characteristic of GSD 0, III, VI, and IX. ${ }^{54}$ Other biochemical characteristics that help to distinguish between these disorders are elevated uric acid and lactate levels in GSD I, whereas these are typically normal in GSD III. At the time of diagnosis, serum concentration of hepatic transaminase (aspartate aminotransferase and alanine aminotransferase) are increased in GSD I and often return to normal or near-normal levels with appropriate treatment. By contrast, serum aspartate aminotransferase and alanine aminotransferase levels are typically higher in GSD III, VI, and IX, and increased levels tend to persist despite treatment. Although elevated transaminase levels and hepatomegaly are common to many primary liver diseases and other metabolic disorders, hypoglycemia is distinctly uncommon until the development of end-stage liver disease for most disorders, except GSDs ${ }^{67,68}$ and disorders of fructose metabolism. An increase in creatine phosphokinase is also often noted in GSD IIIa due to involvement of skeletal and cardiac muscle; however, a normal creatine phosphokinase concentration does not rule out muscle involvement. Whereas patients with GSD VI and GSD IX are usually reported to be relatively mildly affected, some patients are more severely affected and closely resemble patients with GSD III.

Hypoglycemia and ketosis are not typical features of GSD IV. In this disorder, liver dysfunction that progresses to liver cirrhosis is a typical clinical feature. Hypoglycemia is a late finding and is typically only observed in the setting of liver failure. In GSD IV, abnormally structured glycogen resembling plant-like fibers (amylopectin) accumulates in the liver.

Fructose-1,6-bisphosphatase deficiency, ${ }^{69-71}$ a disorder of gluconeogenesis, and Fanconi-Bickel syndrome (GSD XI) ${ }^{72-75}$ both have some features that may be confused with GSD I (Table 1). Because of severe hepatomegaly, lysosomal storage disorders such as Gaucher disease and Niemann-Pick type B disease may initially be confused with GSD I. In both these storage diseases, however, there is striking splenomegaly, which is an important distinguishing feature, and hypoglycemia does not occur. ${ }^{68}$

\section{Clinical and laboratory evaluation}

GSD I most commonly presents as hypoglycemia and/or hepatomegaly in infants. ${ }^{76} \mathrm{~A}$ blood sample drawn at the time of hypoglycemia ("critical sample") is useful in evaluating the various metabolic and endocrine causes of hypoglycemia. The presence of hepatomegaly with hypoglycemia should prompt a workup that includes measurement of BG, lactate, uric acid, hepatic profile including liver function tests, cholesterol, triglycerides, basic chemistry panel, creatine kinase, complete blood cell count with manual differential white cell count, plasma total and free carnitine, acylcarnitine profile, plasma amino acids, $\beta$-hydroxybutyrate, urinalysis, urinary $\mathrm{Hex}_{4}$, and urine organic acids. It should be noted that the pattern of increased low-density lipoprotein cholesterol, decreased high-density lipoprotein cholesterol, and increased triglycerides seen in GSD I is similar to the lipid profile observed in patients with hyperlipidemia type II. In the absence of significant hepatomegaly, blood measurement of lactate, uric acid, triglycerides, and cholesterol, in addition to insulin, growth hormone, and cortisol levels, is recommended to rule out GSD I in patients with hypoglycemia. Neonates and children with GSD I who have mild hepatomegaly may be mistakenly diagnosed and treated for growth hormone deficiency.

In addition, when working up newborns or young infants for hypoglycemia, results of newborn screening (when available) should be checked because fatty acid oxidation disorders and galactosemia (included in standard newborn screening panels) must be considered in the differential diagnosis. Patients with GSD I have significant lactic acidosis during episodes of hypoglycemia with values that may be variable but that are usually $10 \mathrm{mmol} / \mathrm{l}$ or more. If there is a concern about blood lactate levels due to use of a tourniquet to obtain the blood sample, then examination of the basic metabolic panel will provide supportive evidence of a high anion gap metabolic acidosis due to lactic acidosis. The combination of hypoglycemia, lactic acidosis, hypercholesterolemia, hypertriglyceridemia, and hyperuricemia is strongly suggestive of the diagnosis of GSD Ia.

The presence of neutropenia is suggestive of GSD Ib; however, it is prudent to remember that neutrophil counts may be normal during the first 2 years of life.

The clinical and laboratory evaluation should be sufficient to suggest the correct diagnosis, which can be confirmed by noninvasive molecular genetic testing either by targeted mutation analysis based on the patient's ethnic background or by comprehensive gene sequencing.

\section{DIAGNOSTIC TESTING}

\section{Laboratory}

Initial laboratory findings that are consistent with GSD I include hypoglycemia, lactic acidosis, hyperuricemia, hypercholesterolemia, hypertriglyceridemia, and, in GSD type Ib, abnormalities in neutrophils. In addition, some patients may have been evaluated for other causes of hypoglycemia with glucagon stimulation. A glucagon stimulation test may lead to worsening of the metabolic acidosis in GSD I and therefore is not recommended to make the diagnosis of GSD I. If it is performed, very close monitoring is required due to the risk of acute acidosis and decompensation. In GSD I there will be a significant increase in blood lactate but little or no increase in BG concentration. 
Table 1 Differential diagnosis of GSD I

\section{Disorder}

GSD type 0 (glycogen

synthase deficiency)

GSD III (glycogen debrancher enzyme deficiency)
Similarity with GSD I

Fasting hypoglycemia

Hepatomegaly, fasting hypoglycemia, $\uparrow$ AST and $A L T,{ }^{a}$ hyperlipidemia

GSD IV (branching enzyme
deficiency)
GSD VI (hepatic
phosphorylase deficiency)

GSD IX (hepatic form of phosphorylase kinase deficiency)

\begin{tabular}{|c|}
\hline $\begin{array}{l}\text { GSD IX (hepatic form of } \\
\text { phosphorylase kinase } \\
\text { deficiency) }\end{array}$ \\
\hline $\begin{array}{l}\text { GSD XI (Fanconi-Bickel } \\
\text { syndrome due to GLUT } 2 \\
\text { deficiency) }\end{array}$ \\
\hline $\begin{array}{l}\text { Disorders of gluconeogenesis } \\
\text { (e.g., fructose-1,6- } \\
\text { bisphosphatase deficiency) }\end{array}$ \\
\hline $\begin{array}{l}\text { Primary liver disease (e.g., } \\
\alpha \text {-1-antitrypsin, hepatitis) }\end{array}$ \\
\hline $\begin{array}{l}\text { Other storage (metabolic) } \\
\text { diseases (Niemann-Pick B } \\
\text { disease, Gaucher disease) }\end{array}$ \\
\hline $\begin{array}{l}\text { Hereditary fructose } \\
\text { intolerance }\end{array}$ \\
\hline
\end{tabular}

Hepatomegaly, $\uparrow$ AST and ALT, a prolonged PT and low albumin in advanced stage of disease

Hepatomegaly, fasting hypoglycemia, $\uparrow$ AST and ALT, ${ }^{\text {}}$ hyperlipidemia

Hepatomegaly, fasting hypoglycemia, $\uparrow$ AST and ALT, ${ }^{a}$ hyperlipidemia; some rare patients have a proximal renal tubular dysfunction (X-linked form)

Hepatomegaly, fasting hypoglycemia and ketosis, $\uparrow$ AST and ALT, a Fanconi-like renal tubular dysfunction (glucosuria, proteinuria, phosphaturia, generalized aminoaciduria)

Hepatomegaly, fasting hypoglycemia and hyperlacticacidemia, $\uparrow$ uric acid, AST, and ALT

Hepatomegaly, $\uparrow$ AST and ALT ${ }^{\mathrm{a}}$

Hepatomegaly and splenomegaly, growth failure, hyperlipidemia

Hepatomegaly, $\uparrow$ AST and ALT ${ }^{\mathrm{a}}$

\section{Distinguishing features}

Absence of hepatomegaly; postprandial hyperglycemia, hyperalaninemia and hyperlactatemia; fasting ketosis

Hypoglycemia is usually less severe, but the patient may have more severe ketosis and absence of hyperlactatemia and hyperuricemia; $\uparrow$ AST, ALT usually higher (may be $>500 \mathrm{U} /$ )); cardiac and skeletal muscle involvement with $\uparrow$ CK concentrations in GSD IIla; normal blood lactate and uric acid

Lack of hypoglycemia until end-stage liver disease; PT commonly prolonged in GSD IV; increased GGT

Hypoglycemia usually occurs only during fasting and is associated with hyperketosis; GSD VI can be less severe, however, in some patients there is significant hypoglycemia; blood lactate is normal but there can be postprandial elevations.

Hypoglycemia is typically less severe, usually occurs only during fasting, and is associated with hyperketosis; blood lactate is normal; but there can be postprandial elevations metabolic acidosis is rare; some patients develop liver fibrosis which can progress to cirrhosis in rarer cases

Postprandial hyperglycemia; gastrointestinal symptoms (chronic diarrhea from carbohydrate malabsorption); hypophosphatemic rickets; significant short stature

Hypoglycemia after more prolonged (e.g., overnight) fasting or during intercurrent illness with reduced carbohydrate intake

Lack of fasting hypoglycemia and hyperlacticacidemia

Lack of fasting hypoglycemia, significant splenomegaly; storage cells characteristic of the disease, other features such as bone and pulmonary involvement

Gastrointestinal symptoms, long-term liver and kidney damage, prolonged PT, hypoalbuminemia, elevation of bilirubin, and proximal tubular dysfunction; hypoglycemia provoked by fructose intake; improvement of symptoms with fructose restriction

ALT, alanine aminotransferase; AST, aspartate aminotransferase; CK, creatine kinase; GGT, gamma-glutamyl transpeptidase; GLUT 2, glucose transporter 2; GSD, glycogen storage disease; PT, prothrombin time.

aIn GSD I, increased AST and ALT is usually most pronounced in the untreated or inadequately treated state and tends to normalize with appropriate treatment.

Biochemical analysis of liver sample. Hepatomegaly often leads gastroenterologists to perform a biopsy of the liver to differentiate among the diverse etiologies of hepatomegaly. It should be emphasized that biopsies are not necessary when GSD is suspected, because gene sequencing is now available for individual disorders as well as panels of relevant genes. Biopsies should lead to a definitive diagnosis in most cases but are critically dependent on correct processing of the tissue. Tissues should be processed for light microscopy and electron microscopy and also should be snap-frozen $(\sim 15 \mathrm{mg})$ in the operating room in liquid nitrogen for biochemical analysis. Usually $30-40 \mathrm{mg}$ of tissue or four cores of liver tissue are required for all the studies necessary to make a definitive diagnosis. In the United States, reliable enzymatic analysis is available on frozen liver biopsy samples.

Liver histology can help differentiate GSD I from other hepatic forms of GSD. Histopathological findings of the liver in GSD I include distention of the liver cells by glycogen and fat and the finding that glycogen is uniformly distributed..$^{77}$ The amount of glycogen accumulation may be normal or only modestly increased. Lipid vacuoles are large and numerous. ${ }^{77}$ By contrast, in most patients with GSD III, the liver biopsy demonstrates a vacuolar accumulation of non-membrane bound glycogen primarily located in the cytoplasm. Lipid vacuoles are far less numerous in GSD III than in GSD I. The presence of fibrosis, ranging from minimal periportal fibrosis to micronodular cirrhosis, occurs in GSD III, GSD VI, and GSD IX but not in GSD I. ${ }^{18,77,78}$ Periportal fibrosis is a very early finding in GSD III. In both GSD I and GSD III, the stored material is within the cytoplasm, periodic acid schiff positive, and diastase sensitive.

In GSD I, the total glycogen content is much lower than in GSD III, GSD IV, GSD VI, and GSD IX. Clinical assays measure overall G6Pase enzyme activity in liver tissue samples (see description above). Normal G6Pase enzyme activity level in liver is $3.50 \pm 0.8 \mu \mathrm{mol} / \mathrm{min} / \mathrm{g}$ tissue. In most individuals with GSD Ia, the G6Pase enzyme activity is less than $10 \%$ of normal. However, in rare affected individuals with milder clinical manifestations, the G6Pase enzyme activity can be higher (>1.0 
and $<2.7 \mu \mathrm{mol} / \mathrm{min} / \mathrm{g}$ tissue). G6P translocase activity in vitro is difficult to measure in frozen liver biopsy specimens; fresh (unfrozen) liver biopsy tissue is needed to assay enzyme activity accurately. As a result, most clinical diagnostic laboratories do not offer enzyme activity testing for GSD Ib.

Molecular genetic testing. Noninvasive molecular genetic testing through full gene sequencing of the G6PC (GSD Ia) and SLC37A4 (GSD Ib) genes can be used for confirming the diagnosis. ${ }^{79}$ Mutations in the G6PC gene are responsible for $\sim 80 \%$ of GSD I cases, and mutations in SLC37A4 gene are responsible for $\sim 20 \%$ of GSD I cases. Although full gene sequencing for both of these genes is available for clinical testing, targeted mutation analysis can be helpful in some ethnic groups. Testing for specific common mutations can identify up to $100 \%$ of affected individuals, depending on the ethnic group. $7,12,16,17$

Some examples of the common mutations seen in GSD Ia are listed in Table 2.

Some common mutations seen in GSD Ib are listed in Table 3.

Sequence analysis. Although sequence analysis of G6PC can detect up to $100 \%$ of affected individuals in some homogeneous populations, ${ }^{14}$ in mixed populations (e.g., in the United States) the detection rate can be lower $(\sim 94 \%)$, as indicated by some individuals with clinically and enzymatically confirmed GSD Ia for whom only one mutation can be detected. It is possible that these individuals carry large deletion mutations of one or more exons, introns, or the whole gene that are unlikely to be detected by current sequencing methods. Mutations in the promoter region will also be missed by standard sequence analysis.

Similarly, full gene sequence analysis of SLC37A detects mutations in up to $100 \%$ of affected individuals in some homogeneous populations, ${ }^{7,12,79-81}$ but in mixed populations the detection frequency could be lower because both mutations may not be detected in some individuals even though the clinical phenotype is consistent with GSD Ib.

Pathologic allelic variants. At present, 86 disease-causing mutations have been reported in the G6PC gene (GSD Ia) and 82 disease-causing mutations have been reported in the SLC37A4 gene (GSD Ib). The reported mutations are scattered throughout these genes. Some additional, but as yet unreported, mutations have been identified through clinical testing of known patients from various ethnic groups. The mutations identified include missense and nonsense mutations, small deletions and insertions resulting in frameshifts, splice-site mutations, and rare gene rearrangements. ${ }^{7,12,17,79,80,82}$

Deletion/duplication analysis. Large multiexon deletion/ duplications in the G6PC and SLC37A4 genes cannot be detected by sequence analysis. Although the exact frequency of exonic or multiexonic deletions is not known, very few such mutations have been reported in either of these genes ${ }^{83}$ (unpublished clinical laboratory observations). A variety of methods, including quantitative PCR, long-range PCR, multiplex ligation-dependent probe amplification, and targeted array (gene/segment-specific), may be used for deletion/ duplication analysis. Comparative genomic hybridization analysis that detects deletions/duplications across the genome may also include these genes/segments.

\section{Testing strategy for GSD I}

Mutation analysis is the first choice for diagnosis if GSD I is suspected (Box 1). Complete G6PC sequencing is usually performed first, unless neutropenia is present. If snap-frozen liver biopsy tissue is available to confirm the diagnosis, it can be analyzed for G6Pase enzymatic activity. Deficient enzyme activity confirms the diagnosis of GSD Ia.

Table 2 Common mutations seen in GSD la

\begin{tabular}{|c|c|c|c|c|}
\hline cDNA change $^{a}$ & Old cDNA nomenclature ${ }^{b}$ & Amino acid change ${ }^{a}$ & Ethnicity & References \\
\hline c. $247 C>T$ & C326T & p.Arg83Cys & Caucasian (32\%), Jewish (96\%) & $4,8,9,12,15$ \\
\hline c. $248 \mathrm{G}>\mathrm{A}$ & G327A & p.Arg83His & Chinese $(38 \%)$ & $9,12,202$ \\
\hline c.378_379dupTA & 459insTA & p.Tyr128Thrfs*3 & Hispanic (50\%) & $4,12,13$ \\
\hline c. $648 \mathrm{G}>\mathrm{T}$ & G727T & p.Leu216Leu; creates new splice site & Japanese (85-88\%), Chinese (36-40\%) & $9-12$ \\
\hline c. $1039 C>T$ & $1118 C>T$ & p. $G \ln 347^{*}$ & Caucasian $(21 \%)$ & $7,9,12,14$ \\
\hline
\end{tabular}

CDNA, complementary DNA; GSD, glycogen storage disease.

aNomenclature based on the recommendation of the Human Genome Variation Society (http://www.hgvs.org). ${ }^{\text {E} E a r l y ~ r e p o r t s ~ o f ~ m u t a t i o n s ~ i n ~ G 6 P C ~ d e s i g n a t e d ~ t h e ~}$ transcription start site as +1 (ref. 4).

Table 3 Common mutations seen in GSD lb

\begin{tabular}{|c|c|c|c|c|}
\hline cDNA change $^{a}$ & Old nomenclature ${ }^{b}$ & Amino acid change ${ }^{a}$ & Ethnicity & References \\
\hline c.352T>C & $512 \mathrm{~T}>\mathrm{C}$ & p.Trp118Arg & Japanese (37-50\%) & $9,12,80,81,20^{3}$ \\
\hline c. $1015 G>T$ & $1184 \mathrm{G}>\mathrm{T}$ & p.Gly339Cys & Mixed Caucasian (19-21\%), German (29\%) & $9,12,20^{4}$ \\
\hline c. 1042_1043delCT & $1211 \mathrm{delCT}$ & p.Leu348Valfs*53 & Mixed Caucasian (27-31\%), German 32\% & $7,9,12,79,11^{4}$ \\
\hline
\end{tabular}

CDNA, complementary DNA; GSD, glycogen storage disease.

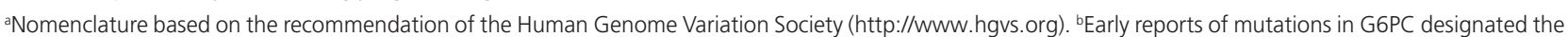
transcription start site as +1 (ref. 205) 
Box 1 Laboratory diagnostic testing recommendations

- Blood/plasma hypoglycemia, lactic acidosis, hypercholesterolemia, hypertriglyceridemia, and hyperuricemia are consistent with GSD type I.

- Neutropenia suggests GSD Ib; however, neutropenia can be seen in GSD la also. Neutrophil counts may be normal in GSD lb during the first years of life.

- Diagnosis should be confirmed by full gene sequencing of the G6PC (GSD la) and SLC37A4 (GSD lb) genes.

- If liver biopsy is performed, histology typically shows fat and glycogen in hepatocytes without fibrosis. Glycogen content is mildly increased as compared with that seen in other liver GSDs (especially GSD III and GSD IX). Diagnosis is often made by measuring G6Pase enzyme activity on a piece of snap-frozen liver biopsy tissue; however, G6Pase enzyme activity on a piece of snap-frozen liver biopsy tissue will not detect GSD lb.

- Targeted mutation analysis is useful for prenatal diagnosis and carrier testing for patients with known private family mutations and may be useful when knowledge of common mutations for specific ethnic groups is available.

\section{OVERVIEW OF MANAGEMENT}

GSD I is a multisystem disorder that is best managed by a multidisciplinary team led by a physician with expertise in managing this disorder; this physician-who may be a metabolic disease specialist/biochemical geneticist, endocrinologist, or hepatologist-coordinates the patient's care together with a metabolic dietician. Other specialists required to manage specific manifestations of the disease include a nephrologist, a hepatologist, a hematologist, a genetic counselor, and a cardiologist. Transplant specialists are consulted when indications for liver and/or kidney disease arise.

All specialists involved in the care of an individual with GSD I should have an understanding of the disease, its protean manifestations, and its unique challenges, including the psychological and emotional impacts of this disease on patients and families. ${ }^{84}$

\section{GASTROINTESTINAL/NUTRITIONAL ASPECTS}

The defect of G6Pase and translocase greatly impacts the nutrition status of those with GSD I. Nutrition therapy for GSD Ia and GSD Ib is the same, but those with GSD Ib may require further dietary intervention related to the consequences of neutropenia such as Crohn disease-like enterocolitis.

\section{Infants and young children}

Hypoglycemia - the primary concern in infants and young children with GSD I-permeates all aspects of their diet and lifestyle (although in rare cases older children or adults with GSD I may present without hypoglycemia) (see Box 2). It is critical that the initial nutrition assessment be broad, not only evaluating the child's intake but also documenting sleep patterns, signs and symptoms of hypoglycemia, the timing of feedings, BG records, gastrointestinal disturbances, and vitamin/mineral supplementation. Children who are fed frequently from birth may not exhibit obvious signs of hypoglycemia until they sleep through the night or have an illness and fast for an extended period of time. A child with an undiagnosed or improperly treated case may have BG levels less than $40 \mathrm{mg} / \mathrm{dl}$ (normal: $70-100 \mathrm{mg} / \mathrm{dl}$ or 3.9-5.6 mmol/l) even after a short fast of 3-4 hours. ${ }^{85,86}$ Early diagnosis reduces the risk of prolonged hypoglycemia, which, untreated, may cause developmental and physical delay, seizures with or without cerebral damage, and even death. Once a diagnosis is made and nutrition therapy is implemented, close BG monitoring and other laboratory parameters must continue as the child grows and nutritional needs change. Without frequent BG monitoring, asymptomatic low BG levels result in suboptimal control, which further inhibits normal growth, development, and overall metabolic control. The aim should be to keep $B G \geq 70$ and to avoid rapid glucose fluxes.

Nutrition therapy. Recurrent hypoglycemia causes lactic acidosis, hepatomegaly, hypertriglyceridemia, hyperuricemia, and failure to thrive in the young child. Thus, avoidance of fasting is the first line of treatment in GSD I. To prevent hypoglycemia, small frequent feedings high in complex carbohydrates (preferably those higher in fiber) are evenly distributed over 24 hours.

In general, the nutrient composition of the diet is $60-70 \%$ calories from carbohydrates, $10-15 \%$ calories from protein (to provide the daily recommended intake), and the remaining calories from fat ( $<30 \%$ for children older than 2 years). . $^{84,87-89}$ As a result of the deficiency of the G6Pase enzyme, fructose and galactose are not metabolized to glucose-6-phosphate, which further contributes to the biochemical abnormalities. ${ }^{90,91}$ There is no consensus regarding the restriction of these two sugars in the diet, but sucrose (fructose and glucose) and lactose (galactose and glucose) are often limited or avoided. ${ }^{89}$ Limiting these sugars reduces or completely eliminates sugar, fruit, juice, dairy, and foods that contain these products from the diet. Careful assessment and supplementation of micronutrients is required to avoid nutrient deficiencies.

\section{Infants}

Formulas and enteral feedings. In infancy, a soy-based, sugarfree formula or a formula that is free of sucrose, fructose, and lactose is fed on demand every 2-3 hours. Once the infant is able to sleep longer than 3-4 hours at a time, several decisions must be made to avoid hypoglycemia during the overnight fast. One option is to continue to wake the infant every 3-4 hours to monitor BG and offer feedings. Another option is to use overnight gastric feedings (OGFs). Due to the life-threatening risks of severe hypoglycemia causing seizures, permanent brain damage, and even death in GSD I, it is recommended that the parents (and/or child, when older) be trained in inserting a nasogastric (NG) tube or that a G-tube be surgically placed so that there is always access to treat for hypoglycemia, especially 
during times of illness or refusal to eat. For patients with GSD Ib and neutropenia, a G-tube may not be a good option because of the risk for recurrent infections at the surgical site. If a child has neutropenia, a G-tube should be placed only if granulocyte colony-stimulating factor (G-CSF) (Neupogen) is being administered. The formula may be offered every 4 hours by mouth and/or by tube, or the formula may be infused continually at a rate to provide adequate glucose to maintain the BG level at more than $70 \mathrm{mg} / \mathrm{dl}$ or $4 \mathrm{mmol} / \mathrm{l}$. Formulas for the OGF may be the same infant formula the child uses during the day (should be sucrose free, fructose free, and lactose free) or it may be an elemental formula that has a higher percentage of carbohydrates. Infusing glucose may be an option in some cases. Feeding regimens are determined case by case. In general, the rate of the continuous tube feeding is calculated to provide a glucose infusion rate of $8-10 \mathrm{mg}$ glucose $/ \mathrm{kg} / \mathrm{min}$ during infancy and $4-8 \mathrm{mg}$ glucose $/ \mathrm{kg} / \mathrm{min}$ in older children. ${ }^{92-94}$ Adjustments of the rate of the tube feeding are made based on BG monitoring so that optimal control is achieved. When the tube feeding is completed each morning, there will still be high circulating insulin levels. Thus, it is important to feed the infant immediately after discontinuing tube feedings to avoid a rapid decrease in glucose (it may be beneficial to feed first and wait $30 \mathrm{~min}$ before disconnecting glucose). OGFs are not without fault or risks. There have been reports of pump failures and occluded or disconnected tubing preventing the formula from being infused and leading to hypoglycemia, seizures, and even death. ${ }^{95}$ Safety precautions such as bed-wetting devices (to detect formula spilling onto the bed), infusion pump alarms, safety adapters, connectors, and tape for tubing should be advised.

Introducing solid food. Solid food is introduced along the normal time line between 4 and 6 months of age. Infant cereals are followed by vegetables and then by meat. Fruits, juice, and other sucrose-containing, fructose-containing, and lactosecontaining foods are limited or omitted. Spoon-feeding, drinking from a cup, and the introduction of table foods should follow the normal feeding progression in order to prevent longterm feeding disorders. Any delays in this progression should be addressed immediately (see later section on feeding issues).

\section{The young child}

Restricting fruit, juice, and dairy foods impacts two entire food groups and renders the diet inadequate. In GSD I, a complete multivitamin with minerals is essential. If a sugar-free soybased milk that is fortified with calcium and vitamin $\mathrm{D}$ is not included, then calcium with vitamin D supplements are also essential. Without appropriate supplements, these children are at risk for a variety of nutritional deficiencies. ${ }^{96}$ In a recent study, $61.5 \%$ of those with GSD I who were tested for $25-\mathrm{OH}$-vitamin $\mathrm{D}$ levels had insufficient levels $(<30 \mathrm{ng} / \mathrm{ml})$. These levels were low despite the subject's reports of taking their prescribed supplements. ${ }^{87}$ Osteoporosis is a known long-term consequence of GSD I. Although the cause may be multifactorial, optimal nutrition at a young age can only help prevent or delay some of the long-term consequences of the disease. Therefore, the focus of the diet (Table 4) must go beyond simply preventing and treating hypoglycemia. ${ }^{97}$

Cornstarch. Raw cornstarch (CS) has been used for the treatment of hypoglycemia in GSD I since the early 1980 s. $^{98}$ There is no consensus regarding the age at which CS therapy should be initiated, but a trial is often introduced between 6 months and 1 year of age. Amylase is needed for the digestion of CS; this enzyme may or may not be fully present until 2 years of age. Starting with a small dose of raw, uncooked CS and gradually increasing the dose to the goal may help improve tolerance. Side effects of CS may include gas, bloating, and diarrhea, but in some cases the symptoms may subside after 2 weeks of therapy. ${ }^{86}$ Those with GSD Ib often have worse gastrointestinal issues and may be diagnosed with a Crohn disease-like colitis.99,100 In some cases, pancrelipase (lipase, protease, and amylase) has been used in conjunction with CS therapy to reduce the side effects, but routine usage is not recommended ${ }^{98} \mathrm{CS}$ is digested slowly, providing a steady release of glucose, which allows more stable glucose levels over a longer period of time as compared with other sources of carbohydrates..$^{98,101}$

General guidelines for dosing CS include $1.6 \mathrm{~g}$ of CS per kilogram of body weight (ideal body weight) every 3-4 hours for young children, and $1.7-2.5 \mathrm{~g} \mathrm{CS} / \mathrm{kg}$ every $4-5$ hours (sometimes 6 hours) for older children, adolescents, and adults. Some adults may eventually only require one dose of CS at bedtime to maintain their BG at more than $70 \mathrm{mg} / \mathrm{dl}$ or $4 \mathrm{mmol} / \mathrm{l}$ and to maintain their lactates at less than $2 \mathrm{mmol} / \mathrm{l}$ through the night. ${ }^{98}$ Given these variations in how often dosing should occur in order to maximize metabolic control including BG, cholesterol, triglycerides, and liver enzymes, and given a limited evidence base related to CS dosing and metabolic control, it is important for adolescents and adults to continue to check BG and lactate levels regardless of how stable they feel personally.

Argo (Summit, IL; http://www.argostarch.com) brand CS, by patient report, is the preferred brand in the United States in terms of both taste and sustainability. Other brands should be used with caution, and randomly switching between brands is not recommended. A modified CS, Glycosade, is available in Europe and the United States for overnight treatment. ${ }^{97}$ Because data are limited with regard to long-term use of Glycosade, the new therapy should be used only with close monitoring of markers of metabolic control. As with any changes to CS brand or dose, changes should be made under the supervision of the metabolic team with frequent BG monitoring.

CS can be mixed in sucrose-free, fructose-free, lactose-free infant formula, sugar-free soy milk, sugar-free drinks, and/or water. In previous studies, mixing CS with lemonade or hot water or taking high doses of vitamin $\mathrm{C}$ resulted in a sharp increase in BG levels, followed by a rapid decline. It was speculated that the heating process and the ascorbic acid disrupted the starch granules, rendering the CS less effective. ${ }^{98,101}$ Patients with low citrate who are prescribed Bicitra have become hypoglycemic when mixing their Bicitra with their CS beverage. The 
Table 4 Foods allowed and foods not allowed in GSD I

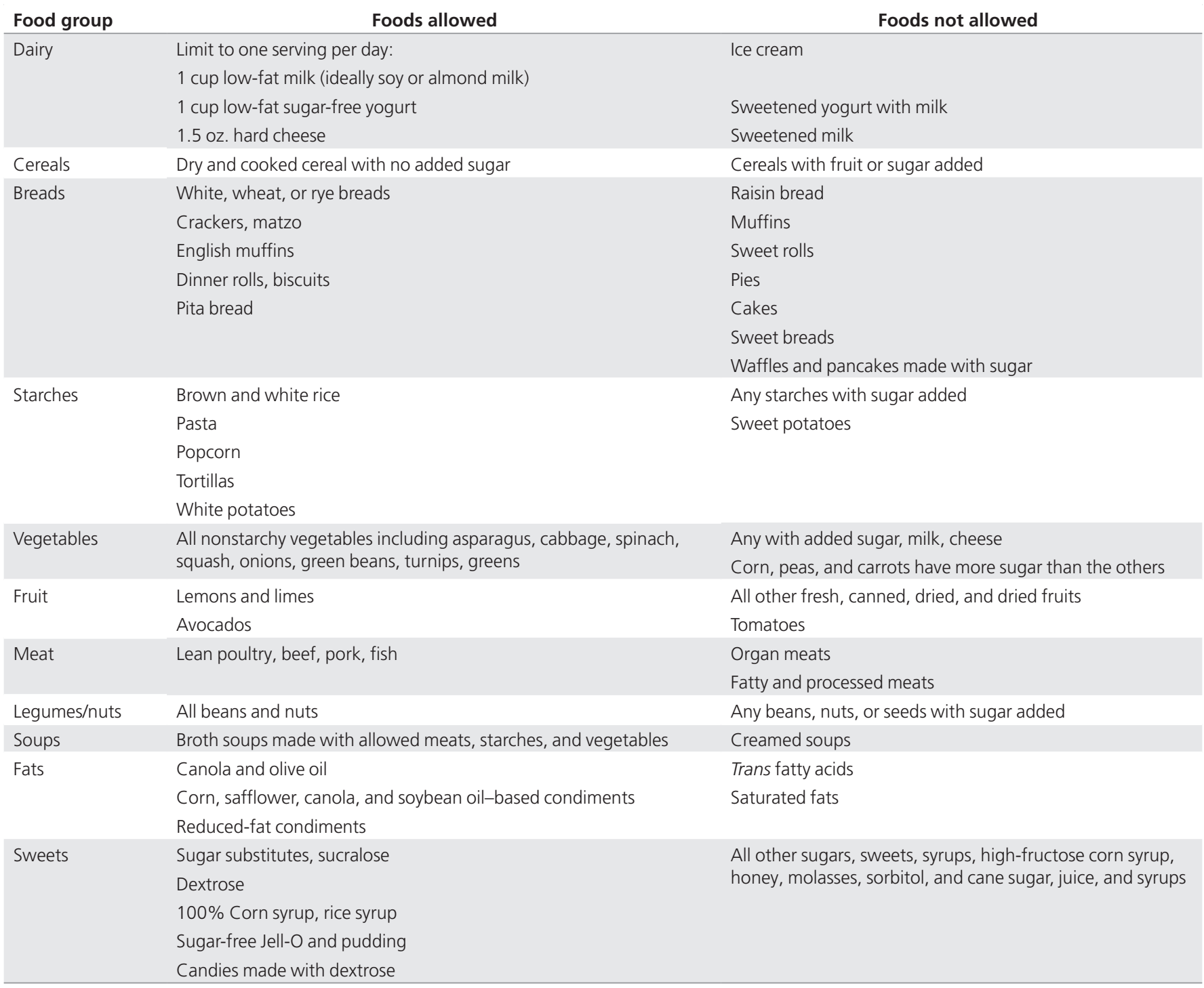

GSD, glycogen storage disease.

Data from ref. 87.

mechanism is likely similar to that described above for lemonade. Until further studies are available to investigate this mechanism, patients should not mix Bicitra with their CS drink.

Ideally, the CS dose should be weighed on a gram scale. When a scale is not available, the dose may be translated into tablespoons. One level tablespoon of CS weighs approximately $8 \mathrm{~g}$. The dilution is approximately $1 \mathrm{~g}$ of CS to $2-3 \mathrm{ml}$ of fluid. The amount of fluid can be adjusted based on preference or tolerance. ${ }^{101}$ Similar dilutions of $3 \mathrm{~g}$ CS to $10 \mathrm{ml}$ fluid have also been suggested ${ }^{89}$ If gastrointestinal disturbances occur, increasing the liquid may be beneficial. As with the OGF, CS therapy also has its limitations. Missed CS doses because of failure of alarm clocks or sleeping through an alarm can lead to hypoglycemia, seizure, and even death. ${ }^{95}$ The use of battery-operated alarm clocks, setting two alarm clocks, and keeping the alarm clock out of reach to avoid rolling over and turning it off should be advised. Parents may need to alternate nightly duties to avoid sleep deprivation that can lead to lapses.

\section{BG monitoring}

BG monitoring is essential for well-controlled GSD. Frequent BG monitoring is needed to establish the initial diet prescription and then should occur randomly to avoid asymptomatic hypoglycemia. BG testing should be documented before each clinic visit so that diet, CS intake, and OGFs can be adjusted. A detailed record noting the time, the BG level, and all foods, CS, and beverages consumed should be provided to the clinic dietitian. The BG levels should be checked before meals, before CS intake, and/or before and after exercise for 2-3 days before the clinic visit. When the CS dose is changed, BG levels should be checked after 4 hours and then at 1-hour intervals to establish 
the duration of effectiveness (how long the dose of CS will maintain the BG level $>70 \mathrm{mg} / \mathrm{dl}$ ). Other changes to routines, school schedules, activities, or those at the onset of illness also require close BG monitoring.

Lactate meter. The use of a portable lactate meter (LactatePro) has been studied and used for the GSD population by at least one group in the United States and in Europe. The lactate meter may be a good supplement to glucose monitoring, especially during times of illness to help prevent acute deterioration, to avoid hospitalization, or to alert the parent that is time to go to the emergency room. The lactate meter has been found more useful in GSD Ia as compared with GSD Ib in one study. ${ }^{102}$

Continuous blood glucose monitoring system. Another tool that is often considered for monitoring and managing BG control in GSD is the continuous glucose monitoring system. ${ }^{103}$ This tool has been used in the diabetic population for more than a decade and, more recently, has been studied in the GSD population. The concern is that the meter may not be as good at detecting low glucose levels, and $20 \%$ of hypoglycemia occurrences were missed in one study. However, this may change with the development of new meters. The use of continuous glucose monitoring systems in the home environment under real-life circumstances may provide more realistic data and may show trends more clearly than in measurements made in the hospital setting. The system may also help detect asymptomatic hypoglycemia.

\section{Treating hypoglycemia}

BG levels should be maintained at more than $70 \mathrm{mg} / \mathrm{dl}$. If the BG level is less than $60 \mathrm{mg} / \mathrm{dl}$, then hypoglycemia should be treated. Signs of low glucose may include lethargy, muscle weakness, nausea, irritability, or a sense of lightheadedness or sweating. However, in many instances, patients with GSD I do not experience low glucose symptoms until BG levels become very low $(<60 \mathrm{mg} / \mathrm{dl})$.

Treatment for hypoglycemia is twofold. First, the low BG must be rescued with a quick-acting source of glucose. Then, a snack or CS is given in order to sustain normal BG. Treatment agents include commercially prepared glucose polymers or over-thecounter diabetic glucose tablets and gels. The amount of glucose given is determined based on the glucose delivery rate desired. All people with GSD I should wear a medical alert bracelet because prompt and appropriate treatment is critical in GSD I.

Continual episodes of hypoglycemia indicate an underlying problem. It may be time to adjust the CS dose or schedule. There may be an intercurrent illness or there may be a compliance factor.

\section{Overtreatment}

Parents fearing the known consequences of hypoglycemia may overcompensate by overtreating and overfeeding their child. Parents should be cautioned against overtreatment at each clinic visit, especially if an increased weight trend is noted.
Other complications of overfeeding, including increased glycogen storage, over time can lead to hyperinsulinemia and insulin resistance. ${ }^{97}$ Excess CS or taking CS too close to meal time reduces the appetite at meal time, limiting the intake of nutritious foods, and can result in nutrient deficiencies. ${ }^{96}$ Overtreatment can also lead to worsening lactic acidosis. Increased gastrointestinal disturbances may also result from excess CS. Scheduling CS and balancing meals can be difficult and the metabolic dietitian should work closely with the family early on to avoid the development of feeding issues.

\section{Feeding issues}

With most chronic illnesses that involve dietary treatment, it may be difficult for the family to achieve an appropriate balance. Children may be delayed making the transition from formula to baby food and from baby food to table food. They may be delayed in weaning from the bottle to a cup. The child may be too full from formula and CS and refuse to take solid foods. The metabolic dietitian will need to address these issues by periodically assessing the diet and adjusting the meal and snack schedules, CS doses, meal times, and OGFs. There is a fine balance between maintaining as much normalcy as possible while meeting the goals of the GSD diet, maintaining normal BG levels, and meeting the child's individual nutrient needs for normal growth and development. If a child continues to show signs of difficulty with feeding, the child should be referred to a speech or occupational therapist for a full feeding evaluation. In some cases, if psychosocial issues are apparent, the family may be referred to the clinical social worker or the child may need a full psychological evaluation. ${ }^{104}$

\section{Growth}

It is important to track the height, weight, weight/height ratio, body mass index, and head circumference in patients with GSD I. Changes in growth trends may reflect poor metabolic control. If revisions to the diet, CS, and OGFs do not improve growth, a referral to an endocrinologist may be indicated.

In the older child who has a delayed bone age, the length needs to be corrected accordingly on the growth chart. Otherwise, the child may be misdiagnosed with poor growth.

\section{Diet and pregnancy}

Successful pregnancies in both GSD Ia and GSD Ib have been reported in the literature. ${ }^{48,105-108}$ Planning ahead of time in accordance with the metabolic team to optimize nutrition, including supplements and tight metabolic control before conception, is recommended. Close BG monitoring is required so that diet and CS dosing and frequency can be adjusted. CS requirements typically increase during pregnancy. The metabolic team and a high-risk obstetrics group should coordinate care together. The admission should be planned in advance so an i.v. glucose infusion can be initiated before delivery to maintain normal BG levels. Good metabolic control also decreases the bleeding complications that could occur at the time of labor and delivery if poor metabolic control is a factor (see Hematology section). 


\section{Long-term complications and nutrition implications}

It is important to review vitamin/mineral compliance annually. Those with GSD I are at an increased risk for osteoporosis. Good metabolic control, including adequate nutrients throughout the life span, may help prevent or delay bone loss. DEXA scans and 25-OH vitamin $\mathrm{D}$ are included as part of the standard screening for GSD I.

Gout is another long-term complication of GSD I. Again, diet adherence and good metabolic control from the onset may prevent the high levels of uric acid that can cause gout. For those with a tendency toward gout attacks, a low-purine diet is prescribed in addition to allopurinol. The side effects of allopurinol should be monitored, including hypersensitivity syndrome and Stevens-Johnson syndrome.

\section{Other dietary considerations}

Elevated triglycerides and cholesterol above the normal ranges may persist in some patients with GSD I, despite appropriate dietary treatment. Although effects of hyperlipidemia in GSD I have been studied for decades, there is no consensus regarding the long-term complications or the best treatment for hyperlipidemia in this disorder. Both dietary and pharmacological treatments have been studied, including fibrates, statins, niacin, and fish oil. ${ }^{109,110}$ The effect of medium-chain triglycerides on lowering cholesterol and triglycerides is currently being studied. ${ }^{111,112}$ The use of vitamin $\mathrm{E}$ and its effectiveness in reducing the frequency of infection and improving neutropenia has been reported. ${ }^{113}$

In conclusion, dietary therapy for the treatment of GSD I has improved the long-term outcomes for patients, but, unfortunately, many complications remain. Further studies of dietary practices and alternative dietary treatments are needed to provide consensus for evidence-based guidelines.

\section{Hepatic manifestations}

\section{LIVER ISSUES}

Hepatomegaly in GSD I attributable to fat and glycogen deposition is universal, resulting in a marked steatotic and enlarged liver. ${ }^{114-116}$ Hepatomegaly is more pronounced in the younger child, resulting in abdominal protrusion; however, with age, the liver size tends to decrease. Given that the stored glycogen is normal in structure, liver enzymes are typically normal in GSD I. An elevation of liver enzymes may sometimes be noted early in the disease course, typically around the time of diagnosis. Hepatocellular adenoma (HCA), HCC, hepatoblastoma, focal fatty infiltration, focal fatty sparing, focal nodular hyperplasia, and peliosis hepatis are some of the liver lesions noted in GSD Ia patients. Of these lesions, HCAs are the most common and typically appear in the second or third decade of life; reported frequencies range from 16 to $75 \% .{ }^{115,117}$ However, there are patients who have HCAs at an older age, leading to a diagnosis of GSD I. ${ }^{19}$

\section{Adenoma characterization}

The prevalence of HCAs increases with age in GSD I. Historically, $70-80 \%$ of patients older than 25 years have at least one lesion. Progression in size and/or number of HCAs occurs in $50 \%$ of patients. ${ }^{33}$ The mean age of adenoma presentation is 14.8 years, but HCAs have been reported in younger patients. Adenomas noted in patients with GSD I are different than those that are noted in the general population. GSD Ia patients seem to present with greater numbers of HCAs that are more likely to be in a bilobar distribution than those in the general population. Furthermore, unlike in the general population, there is no gender predisposition in GSD I. One study noted that of 66 HCAs detected by magnetic resonance imaging in 14 patients, 44 lesions were found in 5 patients, with a mean of 5 lesions per GSD I patient. ${ }^{118}$ The general population usually has single, large, encapsulated HCAs, commonly caused by the use of oral contraceptive pills. ${ }^{24}$ In GSD I patients, HCAs are thought to be the result of inadequate metabolic control. A recent study demonstrated decreased adenoma formation in the setting of good metabolic control, and regression of adenomas has occurred

\section{Box 2 Gastrointestinal/nutrition recommendations}

- A metabolic dietitian is an important member of the team. If not available, one should be consulted.

- Maintaining blood glucose levels $\geq 70 \mathrm{mg} / \mathrm{dl}$ is important to achieve good metabolic control. Levels should be kept consistent to avoid hypoglycemia and fluctuations in the blood glucose levels.

- In infants and children:

- Avoid fasting for more than 3-4 hours.

- Offer small, frequent feedings; avoid or limit sucrose, fructose, and galactose (a soy formula such as Prosobee may be used overnight)

- Access via NG or G-tube placement is recommended for emergencies and/or for OGFs; caution with surgical G-tube placement should be taken in GSD lb.

- Monitor blood glucose before feeds.

- Raw, uncooked cornstarch may be introduced between 6 and 12 months of age.

- Continuous gastric feedings may be used overnight.

- In adolescents and adults:

- Avoid fasting for more than 5-6 hours with the use of raw, uncooked cornstarch and/or OGFs; it is important to not change the brand of cornstarch. If changed, then monitoring of BG levels after the change is necessary.

- Plan for small, frequent meals (nutrient distribution: 60-70\% carbohydrates, $10-15 \%$ protein, $<30 \%$ fat); avoid or limit sucrose, fructose, and galactose.

- Regular blood glucose monitoring is needed, especially during periods of growth.

- Multivitamins, calcium, and vitamin D are necessary because of the restricted nature of the diet.

- Both overtreatment and undertreatment are harmful. Overtreatment can result in insulin resistance.

- Good glucose control improves several of the metabolic sequelae of GSD I. 
in some patients after outstanding metabolic control was achieved. ${ }^{118}$ It should be remembered that factors other than metabolic control can play a role in the development of adenomas (see next section). As discussed in the Gynecological/ Obstetric Care section, estrogen-based contraceptives should be avoided when possible.

\section{Hepatocellular carcinoma}

Because patients with GSD I live longer, new long-term complications are being recognized. HCC has been noted in several patients with GSD I.

There are several challenges concerning the diagnosis of HCC in GSD I. The cause for HCC is unclear, but there appears to be an adenoma-to-HCC transformation, rather than HCC arising in normal liver tissue. Because of the abundance of adenomas, biopsy is not an option. There is no effective biomarker because $\alpha$-fetoprotein and carcinoembryonic antigen levels are often normal even in the setting of HCC. No good imaging tool separates HCA from HCC.

Until recently, the genetic makeup of the adenomas from patients with GSD I was not known. However, Kishnani et al. ${ }^{120}$ recently published the findings of chromosomal and genetic alterations in 10 cases of HCA associated with GSD type I using a sensitive genome-wide high-density single-nucleotide polymorphism analysis and mutation analysis of two target genes, HNF1A and CTNNB1. Chromosomal aberrations were identified in $60 \%$ of the HCAs from GSD Ia adenomas, with the most significant chromosomal aberration on chromosome 6 , showing a simultaneous gain of $6 \mathrm{p}$ and loss of $6 \mathrm{q}$. Although loss of $6 \mathrm{q}$ without gain of $6 \mathrm{p}$ was identified in two (non-GSD I HCA) general population HCAs in this study, and simultaneous gain of $6 \mathrm{p}$ and loss of $6 \mathrm{q}$ has been reported in two general population HCAs in a previous report, ${ }^{121}$ the significance of loss of $6 \mathrm{q}$ for HCA development in the general population was inconclusive because the aberration was just one of multiple chromosomal aberrations in these cases. By contrast, the fact that chromosome 6 alterations was the major finding, with minimal changes in other chromosomes in three GSD Ia HCAs, strongly suggests that loss of $6 q$ and/or gain of $6 p$ may be an early event in the liver tumorigenesis in GSD I. It is speculated that GSD I HCA with simultaneous gain of $6 \mathrm{p}$ and loss of $6 \mathrm{q}$ could confer high risk for malignant transformation, implicating genes on chromosome 6 in the transformation of HCA to HCC. Patients with these high-risk aberrations may be good candidates for LT until we have a better understanding of the pathogenesis and other therapeutic targets. These findings also suggest that good metabolic control alone may be insufficient to prevent the development of HCA in some patients with GSD I.

\section{Clinical monitoring}

In the general population, HCAs regress in some patients after the cessation of oral contraceptives. In GSD I, there is some evidence that metabolic control may be a modifier of adenoma formation and progression, ${ }^{118}$ but there are cases in which adenomas occur despite good metabolic control. Whereas most investigators agree that HCAs in GSD Ia patients should be observed for signs of malignancy, the management of concerning lesions is not established. Liver imaging is routinely performed in individuals with GSD I. In children ( $<18$ years), liver ultrasounds can be performed every 12-24 months. With increasing age, computed tomography or magnetic resonance imaging scanning using i.v. contrast should be considered to look for evidence of increasing lesion size, poorly defined margins, or spontaneous hemorrhage. ${ }^{24,33,84,122}$ The use of i.v. contrast to minimize the number of missed lesions is recommended. Laboratory testing should include serum transaminases, creatinine, international normalized ratio (prothrombin time/partial thromboplastin time), albumin, and bilirubin tests every 6 months to yearly to monitor the extent of hepatic damage and to delineate if there is progression of liver disease, especially in the setting of LT. It is also known that $\alpha$-fetoprotein and carcinoembryonic antigen levels do not predict the presence of HCAs or malignant transformation ${ }^{24,117}$ in patients with GSD I (see next section).

Initially, the management of liver adenomas in the GSD I population should be conservative (Box 3). An approach of watchful waiting may be used. There are reports of the use of percutaneous ethanol injection as the initial treatment of enlarging liver adenomas. ${ }^{123}$ However, if there are concerns of malignant transformation or the possibility of life-threatening hemorrhage, adenoma resection is recognized as a therapeutic option. Resection of HCAs suspected of being malignant is an effective intermediate step in the prevention of HCC in GSD Ia patients. As such, adenoma resection may be used as the initial management of lesions suspicious for malignancy in GSD I. A study by Reddy et $\mathrm{al}^{23}$ is the largest single-center study of adenoma resection in GSD Ia patients and the only study comparing clinical outcomes between GSD Ia patients and the general population. In this study it was noted that GSD Ia patients present with a greater burden of adenomatous disease and shorter progression-free survival after resection than the general population. This experience of HCA resection in GSD Ia patients demonstrates that partial hepatectomy is feasible in these patients and is an effective intermediate step in the prevention of HCC until definitive treatment such as a LT. Because of the low numbers, the true risks of partial hepatectomy particular to this population have not been explored.

\section{Indications for LT}

Liver replacement is the ultimate therapy for hepatic metabolic disease. It should be considered for patients with multifocal, growing lesions that do not regress with improved dietary regimens and who do not have evidence of distant metastatic disease.

The first reported LT for GSD I was performed in 1982 (ref 124). Since then, more than 100 children and adults with GSD Ia have undergone liver transplantation in North America with a 1-, 5-, and 10-year survival rate of $82 \%, 76 \%$, and $64 \%$, respectively. ${ }^{125}$ After liver transplantation, all GSD I patients have achieved resolution of their metabolic derangement, including correction of hypoglycemia, lactic acidosis, hyperuricemia, and hyperlipidemia. ${ }^{26}$ Other benefits of transplantation include liberalization of the diet and reduction in the risk of malignancy. ${ }^{42}$ 
However, there are several obstacles to LT in GSD Ia patients. These include uncertainties regarding timing of transplantation, limited organ availability, prospects of worsening renal function with immunosuppression, and fears of poor patient compliance with immunosuppressive medication given a history of faulty adherence to a strict dietary regimen..$^{33,63,122,127} \mathrm{In}$ the United States, and increasingly in other countries, priority for LT is governed by the individual's "model for end-stage liver disease" (MELD) score. This score is calculated using a logarithmic assessment of three objective and reproducible variables, namely total serum bilirubin and creatinine concentrations, and the international normalized ratio. The score may range from as low as 6 to a high of 40. In contrast to the Child's score, which formed the basis of assessment of disease severity, and therefore organ allocation until 2002, the MELD score represents a continuous assessment of liver disease severity. ${ }^{127}$

The primary function of the MELD score is to estimate an individual's mortality risk from liver disease and its complications during the next 90 days: the higher the score, the greater the risk of death. A MELD score of 15-17 is significant in that this is the point at which the mortality risk associated with liver disease and its complications is equivalent to the 1-year mortality associated with complications arising from $\mathrm{LT} .{ }^{128}$ Since the inception of the MELD score as the basis for deceased donor organ allocation, patients with HCC have been granted additional priority.

In GSD I, because the hepatic abnormalities are the result of a single-gene, cell-autonomous defect, there is no possibility of recurrence of primary liver disease within the transplanted allograft.

The most common indication for liver transplantation in GSD I has been hepatic adenomatous disease for removal of potentially premalignant lesions. Other indications have included growth failure and poor metabolic control. ${ }^{22,42}$ Optimal metabolic control appears to normalize growth and minimize the risk of hepatic adenomas, and surgical resection is recommended over transplantation when solitary lesions are present. ${ }^{23,118}$ Thus, in light of the modest mortality risk associated with transplantation, the high rate of complications, and improved prognosis with medical management, routine transplantation is not recommended. Transplantation should be reserved for patients who have not had success with medical management, have a history of recurrent adenomas despite liver resection, have a rapid increase in the size and number of liver adenomas, and are at high risk for liver cancer.

Although the survival rate after transplantation has improved over the past 20 years, complications in the postoperative course remain. Chronic renal failure is a well-documented complication of liver transplantation in GSD Ia, ${ }^{129}$ and some patients with GSD Ia have progressed to renal failure within a few years of transplantation. ${ }^{22,126}$ Of note, nephropathy occurs even in GSD type Ia patients without overt evidence of renal disease at the time of liver transplantation. ${ }^{130}$ The use of nephrotoxic transplant medications has been proposed as one contributing factor. Alternatively, a primary GSD-related nephrotoxic effect may be present because of the untreated condition in the kidney. Postoperative pulmonary hypertension has also been documented in a small number of patients after transplantation.

Although hypoglycemia similarly abates when liver transplantation is performed in GSD Ib, the neutropenia, neutrophil dysfunction, and Crohn disease-like inflammatory bowel disease are variably affected by liver transplantation. G-CSF is still often needed to treat the neutropenia associated with GSD Ib despite normalization of the metabolic profile after liver transplantation because neutropenia is primarily attributable to an intrinsic defect in the neutrophils of GSD Ib patients and is not corrected by LT. ${ }^{126,131}$ Posttransplant immunomodulation may also increase neutropenia risk in GSD Ib patients after

\section{Box 3 Hepatic and hepatic transplant recommendations}

- There should be monitoring for the development of liver adenomas via liver imaging especially after the onset of puberty.

- Adenomas are often multiple. In some situations, there is regression of adenomas noted with good metabolic control. Other genetic factors can play a role in hepatic adenoma development. There is a risk for adenoma to HCC transformation, especially when there is a rapid increase in size or number of adenomas. In the setting of adenomas, routine laboratory testing to include hepatic profile (serum glutamic oxalacetic transaminase, serum glutamic pyruvic transaminase, albumin, bilirubin) should be performed every 6 months. In the setting of consideration of an $\mathrm{LT}$, laboratory testing that includes serum creatinine and international normalized ratio (prothrombin time/partial thromboplastin time) tests, in addition to hepatic profile, should be performed every 6 months.

- $\alpha$-Fetoprotein and chorionic embryonic antigen levels are often normal, even in the setting of HCC, and do not predict hepatocellular adenoma to malignancy transformation.

- Abdominal ultrasound is reasonable in the pediatric population. Abdominal imaging should be performed at baseline and then every 12-24 months.

- Abdominal computed tomography/magnetic resonance imaging with contrast should be performed in older patients or patients within the pediatric age group once adenomas are detected on ultrasound and are to be repeated every 6-12 months or earlier based on laboratory and clinical findings.

- Percutaneous ethanol injections, radiofrequency ablation, and partial liver resection are treatment options for liver adenomas (especially if an increase in size, number, or bleeding is noted). A high suspicion of HCC is needed because no reliable biomarker is currently available for HCA-to-HCC transformation. A sudden increase in size or number, or an increase in vascularity of adenomas, is concerning for HCC transformation.

- Monitoring of the patient's MELD score is critical because it is used to assess the extent of liver disease and for ranking for LT. The latter should be performed at centers with experience in ranking GSD I severity. 
transplantation. ${ }^{132}$ Thus, a lot of caution needs to be exercised when deciding on LT as a treatment option for GSD.

\section{NEPHROLOGY}

Renal manifestations of GSD I appear early in childhood and often go undetected without specific diagnostic evaluation. Glycogen deposition occurs in the kidneys, which typically are large on renal imaging; however, nephromegaly is not sufficient to be readily detected on physical examination. As a result of both the metabolic perturbations that arise and the glycogen accumulation with GSD I, there can be not only proximal and distal renal tubular dysfunction but also progressive glomerular injury that can result in functional renal impairment and even end-stage renal disease requiring renal replacement therapy. Specific interventions aimed at ameliorating or trying to prevent the progression of these renal consequences of GSD I are best commenced early after their presentation to have the best opportunity to alter the course of renal injury.

\section{Proximal and distal tubular dysfunction}

The proximal tubule is the site of a great deal of energy expenditure and G6Pase activity is normally highest. With proximal tubular dysfunction, wasting of bicarbonate, phosphate, glucose, and amino acids can be seen. In GSD I, proximal tubular dysfunction has been ascribed to glycogen accumulation in proximal tubular cells or inability to produce glucose for metabolic needs. In children with poorly controlled GSD I, there tends to be more documentation of aminoaciduria and phosphaturia because these children have such low serum glucose and bicarbonate levels that little tubular reabsorption is required. The other proximal tubular defects improve with effective therapy such as the provision of CS and, as a result, tend not to be seen in most patients receiving treatment to maintain glucose levels. ${ }^{37}$

Along the proximal tubule, there is also sodium-linked reabsorption of calcium and the organic acids such as citrate that can freely cross the glomerular filtration barrier. Up to $90 \%$ of filtered citrate is usually reabsorbed, although citrate reabsorption does tend to decrease during adulthood, likely due to changes in the maximal transport capacity of aging nephrons. The citrate that remains in the urine plays an important role in enhancing the ionic strength of the urine, essentially chelating urinary calcium and helping to prevent its precipitation and the development of nephrolithiasis or nephrocalcinosis. As a result, individuals with low urinary citrate levels are more predisposed to urinary tract calcifications, and such urinary tract calcifications can increase the chances of urinary tract infection or mediate renal parenchymal damage with loss of renal functional reserve.

In normal individuals, urinary citrate excretion exceeds $5 \mathrm{mg} /$ $\mathrm{kg} /$ day or 300 to $400 \mathrm{mg} / \mathrm{g}$ urinary creatinine in spot samples of urine. With GSD I, instead of the usual increasing urinary excretion of citrate with ongoing maturity, there is an actual decrease in citrate excretion that accelerates during adolescence and early adulthood. ${ }^{40}$ Although citraturia is influenced by acid-base homeostatic mechanisms, with increasing urinary citrate excretion seen in systemic alkalosis and decreasing urinary citrate excretion seen in systemic acidosis, metabolic control alone is not responsible for the changes in citrate excretion in GSD I because, even in metabolically well controlled GSD I with normal acid-base status or compensated mild metabolic acidosis without systemic $\mathrm{pH}$ change, there is widespread hypocitraturia. ${ }^{40}$ There has also been speculation that, over time, GSD I patients develop an incomplete distal renal tubular acidosis that may also contribute to the low urinary citrate levels and hypercalciuria. ${ }^{62}$

Glycogen deposition in the proximal tubule does reduce proximal tubular calcium reabsorption and is the likely mechanism for altered urinary calcium levels in GSD I. Normally, urinary calcium excretion is less than $4 \mathrm{mg} / \mathrm{kg} /$ day or less than $0.2 \mathrm{mg} / \mathrm{mg}$ when a random urinary calcium to creatinine ratio is obtained. Hypercalciuria is widespread in prepubertal children with GSD I, and the likelihood for nephrolithiasis and nephrocalcinosis increases with ongoing significant elevation in urinary calcium levels. ${ }^{60,61}$ The combination of hypercalciuria and hypocitraturia enhances the likelihood for urinary calcium precipitation and readily accounts for the high rates of urinary tract calcifications seen in GSD I.

\section{Therapeutic strategies for renal tubular dysfunction}

Oral citrate supplementation will augment citrate excretion, favorably altering the urinary milieu to decrease the chances of urinary calcium precipitation and, as a result, is likely very beneficial in GSD I patients with low urinary citrate levels (Box 4). With citrate supplementation, the aim is to achieve at least $300 \mathrm{mg} / \mathrm{g}$ creatinine on spot urine ratios. In individuals with normal renal function, potassium citrate is preferred over sodium citrate because higher sodium intake is linked to greater urinary calcium excretion. It also can result in systemic hypertension. In young children, liquid potassium citrate preparations are generally well tolerated at an initial dose of $1 \mathrm{mEq} / \mathrm{kg} /$ day divided into three doses, with dose augmentation directed by urinary citrate excretion. In older children and adults, potassium citrate tablets at a dose of $10 \mathrm{mEq}$ three times per day can also be commenced and the dose adjusted as needed. Because the effects of citrate supplementation wane over time, multiple daily doses spread over the waking hours are preferred to maximize the proportion of the day with improved urinary citrate levels. Citrate use should be monitored because it can cause hypertension and lifethreatening hyperkalemia in the setting of renal impairment. Patients should also be monitored for sodium levels.

With hypercalciuria, thiazide diuretics can also be provided as a way to enhance renal reabsorption of filtered calcium and decrease urinary calcium excretion. Especially in GSD I individuals with known urinary tract calcification and ongoing hypercalciuria, thiazide diuretic therapy can be considered. Chlorothiazide is used in young children who require liquid preparations; tablets of hydrochlorothiazide are recommended for older children and adults. The efficacy of therapy can be gauged by interval urinary calcium-to-creatinine ratios. 
This ability to decrease urinary calcium excretion is unique to thiazide diuretics, unlike other classes of diuretics that tend to increase urinary calcium excretion.

Other nonspecific measures to reduce urinary calcium deposition, such as optimizing hydration, maintaining a no-added salt diet, or supplementing magnesium intake, can also be considered on an individual basis as well.

\section{Glomerular injury}

GSD I mediates hemodynamic and structural changes in the kidney that can lead to the development of glomerular injury. The exact mechanisms by which these changes occur are not well understood, but activation of the renin-angiotensin system, prolonged oxidative stress, and profibrotic cytokines such as transforming growth factor- $\beta$ have all been implicated, as well as alterations in renal tubular epithelial cell energy stores related to G6Pase defects. ${ }^{133-136}$ These renal changes occur early, and many children with GSD I will have evidence of glomerular hyperfiltration or elevation in the glomerular filtration rate (GFR) to more than $140 \mathrm{ml} / \mathrm{min} / 1.73 \mathrm{~m}^{2}$ within a few years of life. ${ }^{137}$

These changes in GFR may not be readily detected because they result in serum creatinine levels that are often reported as normal. Many clinicians are less familiar with the concept of GFR and the use of certain validated formulas such as the Bedside Schwartz Equation, which allows the GFR to be estimated in children using the serum creatinine value and the child's height, or the Modification of Diet in Renal Disease Study Equation, which uses serum creatinine, age, gender, and ethnicity to estimate the GFR in adults..$^{138,139}$ These equations have been validated against nuclear medicine isotope GFR assessments and are logistically easier and less costly than the gold standard GFR studies or less accurate older methods of assessing GFR such as timed urinary creatinine clearance studies.

With hyperfiltration, enhanced glomerular blood flow and intraglomerular pressure occur. ${ }^{140}$ Over time, these factors seem to accelerate the normal rate of glomerular obsolescence. As glomeruli become obsolete, fibrosis replaces surface area that previously allowed filtration. Histologically, this injury appears as focal and segmental sclerosis, with a subset of glomeruli demonstrating limited scarring. ${ }^{60}$ As this process continues, scarring progresses and encompasses entire glomeruli with resulting loss of viability of the tubular segments and areas of interstitial fibrosis of those nephrons. As more and more glomeruli are lost to scarring, the overall GFR decreases and there is then an accelerated rate of obsolescence in these remnant glomeruli, creating even more stimuli for further glomerular injury.

Generally, because there is early glomerular scarring, there is the development of microalbuminuria, with urinary albumin-to-creatinine ratios exceeding $30 \mu \mathrm{g}$ albumin/mg creatinine. Over time, microalbuminuria has a tendency to progress to frank proteinuria with urinary protein-to-creatinine ratios exceeding $0.2 \mathrm{mg}$ protein $/ \mathrm{mg}$ creatinine. Chronic proteinuria is thought to exacerbate glomerular injury through induction of chemokines and inflammatory pathways. In GSD I, the development of pathologic proteinuria may be inevitable. In a large European cohort, by age 25 years, more than $50 \%$ of patients had frank proteinuria, with the remainder all demonstrating microalbuminuria. ${ }^{33}$

In GSD I, this initial period of hyperfiltration that leads to microalbuminuria and frank proteinuria does seem to then progress to widespread glomerular scarring and eventual renal dysfunction. Most renal biopsy samples from GSD I patients with frank proteinuria or any decrease in GFR demonstrate focal and segmental sclerosis as the histologic change that precedes the loss of renal function and progression to end-stage renal disease. ${ }^{60,135,137}$

\section{Attenuating hyperfiltration injury}

There have been some data to suggest that metabolic control in GSD I may affect the progression of renal injury. In one study, a significantly smaller proportion of patients with optimal metabolic control of their serum glucose, triglyceride, and uric acid concentrations and urine lactate/creatinine ratios had microalbuminuria or proteinuria than patients with poor control. ${ }^{135}$ Once proteinuria has occurred and there is established renal injury in GSD I, it becomes less clear if metabolic control alone alters the loss of GFR over time, underscoring the importance of using other forms of treatment that may delay the process.

For many years, angiotensin blockade has been used to blunt proteinuria and slow loss of GFR in patients with renal diseases such as diabetes mellitus, in which there is similar hyperfiltration injury. ${ }^{141}$ There are also data showing that GSD I patients treated with angiotensin blockade show improvement in their degree of glomerular hyperfiltration and can demonstrate restored normal rates of GFR for some time. ${ }^{142}$ Angiotensin blockade also seems to slow the tempo of GFR loss once it has started to occur. ${ }^{135}$ Use of either an angiotensin-converting enzyme (ACE) inhibitor or an angiotensin receptor blocker (ARB) medication by itself can be efficacious. In cases in which there is a need for further angiotensin blockade, use of both an $\mathrm{ACE}$ and an ARB can prove synergistic to reduce proteinuria, with no increased rate of hyperkalemia or drug-related renal insufficiency. ${ }^{143}$

Although not yet tested in any systematic fashion in GSD I, the role of initiating angiotensin blockade with the early onset of persistent microalbuminuria seems to be a potential strategy to try to slow the factors that cause accelerated glomerular obsolescence and that ultimately lead to microalbuminuria, proteinuria, and renal insufficiency. ${ }^{142}$ Because these agents tend to be well tolerated, and because many GSD I patients will ultimately receive angiotensin blockade later in their disease course, there seems to be limited risk in considering such an approach as a way to try to alter the natural course of GSDassociated nephropathy.

\section{Routine evaluation and management}

Typical measures to maintain GSD metabolic control are beneficial to general renal health because they help prevent 
acidosis and limit hyperuricemia and hyperlipidemia. Chronic acidosis can predispose to higher urinary calcium excretion and decreased urinary citrate, both problems that already exist in GSD I. Hyperuricemia and hyperlipidemia by themselves have both been implicated in causing or accelerating renal injury.

In patients receiving effective dietary therapy for their GSD I, it is unlikely that there will be diffuse proximal tubular dysfunction. There should be periodic assessment of serum electrolytes, calcium, and phosphate as well as interval measurement of blood urea nitrogen and creatinine levels.

GFR should be estimated from the serum creatinine using a validated formula such as the Bedside Schwartz Equation in children or the Modification of Diet in Renal Disease Equation for adults. Hyperfiltration is present if estimated GFR exceeds $140 \mathrm{ml} / \mathrm{min} / 1.73 \mathrm{~m}^{2}$, and angiotensin blockade should be considered and potential risks/benefits should be discussed with the patient and family given the natural course of hyperfiltration accelerating glomerular obsolescence.

In the event that a patient has not yet begun follow-up with a nephrologist and there is a decrease in estimated GFR to less than $60 \mathrm{ml} / \mathrm{min} / 1.73 \mathrm{~m}^{2}$, referral should be made to help coordinate aspects of necessary care that will arise due to advancing chronic kidney disease and further decrease in GFR.

Screening urinalysis should be performed at intervals on all GSD I patients. The presence of hematuria determined by dipstick should lead to assessment of urinary calcium excretion and ultrasound imaging of the urinary tract for calcifications. Even in the absence of hematuria, renal ultrasound should be performed at intervals to assess kidney size and to assess for evolving nephrocalcinosis or nephrolithiasis. Especially for purposes of screening or for routine follow-up, ultrasound is preferred to other imaging techniques.

Despite good metabolic control, hypocitraturia and hypercalciuria may be common in GSD I and, as a result, urine should be assessed at regular intervals for calcium and citrate excretion even if urinalysis is benign. Spot samples are adequate and easier and quicker to collect than are those of timed urine collection.

With hypocitraturia, citrate supplementation should be considered, especially if there is concomitant hypercalciuria or a history of nephrolithiasis or nephrocalcinosis. With hypercalciuria, there needs to be ongoing good hydration and consideration of thiazide therapy to reduce urinary calcium levels, especially in individuals with known or recurrent urinary tract calcifications.

Urine should also be assessed for microalbuminuria and proteinuria. With a negative screening urinalysis for proteins, urine albuminuria should be quantified by spot albumin-tocreatinine ratio. Dipstick-positive proteinuria should be quantified by urinary protein-to-creatinine ratio. Positive results should be confirmed using a first morning void sample to rule out any orthostatic component.

Persistent microalbuminuria or frank proteinuria warrants initiation of angiotensin blockade despite patients being normotensive. Medications should be adjusted to try to blunt the proteinuria to levels that are normal or as near normal as possible as tolerated without causing postural hypotension or hyperkalemia. Attempts should be made to maintain angiotensin blockade chronically, and medication sequelae should be treated in some fashion so that the angiotensin blockade can be maintained or a different type of angiotensin blockade (ACE vs. ARB) should be attempted.

Because chronic hypertension accelerates renal injury, blood pressure should be maintained in a normal range for adults and at less than the 90th percentile for age, gender, and height for children. If antihypertensive therapy needs to be started, angiotensin blockade with ACE or ARB should be considered as first-line therapy if not already instituted for other reasons. Loop diuretics should be avoided because of the risk of hypercalciuria.

With renal insufficiency, there is decreased production of erythropoietin (EPO) by the kidney and anemia may develop. Patients with GFRs less than $50 \mathrm{ml} / \mathrm{min} / 1.73 \mathrm{~m}^{2}$ are particularly prone to development of anemia attributable to chronic kidney disease. Concomitant clinical factors in GSD patients such as chronic metabolic acidosis, iron deficiency, and bleeding diathesis may potentiate or exacerbate this anemia. In children and adolescents with chronic kidney disease, anemia is linked to impairments in cognitive and developmental gains as well as increased hospitalization rates. Accordingly, when hemoglobin decreases to less than $10 \mathrm{~g} / \mathrm{dl}$, EPO therapy is initiated and titrated to maintain levels between 10 and $12 \mathrm{~g} / \mathrm{dl}$. With adults, there are fewer data to support a specific hemoglobin level under which EPO should be started. As a result, EPO therapy is initiated if there is any evolving symptomatic anemia to prevent the need for blood transfusion. Adults using EPO should avoid hemoglobin levels higher than $12 \mathrm{~g} / \mathrm{dl}$ because these levels may increase the risk for cardiovascular events. Because iron deficiency anemia is common in GSD I, it is prudent to screen both children and adults with chronic renal failure for iron deficiency anemia and replace iron as needed before starting EPO therapy.

Long-term exposure to nephrotoxic medications should also be avoided. This includes use of nonsteroidal anti-inflammatory drugs such as ibuprofen and is especially important if there is any reduction in GFR or if patients have a bleeding diathesis.

With cases of advanced chronic kidney disease and a GFR that declines to less than $15 \mathrm{ml} / \mathrm{min} / 1.73 \mathrm{~m}^{2}$, renal replacement therapy with dialysis or transplantation needs to be considered. Metabolic derangements from ongoing chronic renal insufficiency may exacerbate some of the issues that arise from GSD, making renal transplantation a more attractive therapy. In this case the option of both liver and kidney transplant may be considered.

\section{HEMATOLOGIC ASPECTS}

Hematologic aspects in GSD I include risk for anemia, bleeding diathesis, and neutropenia in GSD Ib.

\section{Anemia in type la}

Anemia is a significant long-term morbidity in individuals with GSD I. In 1994, Talente et al. ${ }^{114}$ reported that $81 \%$ of patients with GSD Ia had anemia in adulthood. 


\section{Box 4 Nephrology recommendations}

- Diagnostic studies should be performed at routine visits to follow renal manifestations of GSD I, including

- Renal ultrasound to assess kidney size and growth, nephrolithiasis, or nephrocalcinosis;

- Urinalysis for hematuria and proteinuria;

- Quantification by spot samples of urinary microalbumin/creatine excretion, citrate, and calcium/creatine excretion;

- Measurement of serum electrolytes, calcium, and phosphate; blood urea nitrogen and serum creatinine with calculation of estimated GFR.

- Consider initiating an ACE inhibitor or ARB with evidence of hyperfiltration (sustained estimated GFR $>140 \mathrm{ml} / \mathrm{min} / 1.73 \mathrm{~m}^{2}$ ).

- Initiate an ACE inhibitor or ARB for persistent microalbuminuria (>30 $\mu \mathrm{g}$ albumin /mg creatinine).

- Initiate an ACE inhibitor or ARB for frank proteinuria $(>0.2 \mathrm{mg}$ protein/mg creatinine).

- Initiate citrate supplementation for hypocitraturia, use of potassium citrate in patients with good renal function, accompanied by careful monitoring of electrolytes. Use extreme caution in the setting of renal failure.

- Consider a thiazide diuretic for hypercalciuria.

- Maintain normal blood pressure for age.

The report was based on an observational study of 32 subjects. Anemia in the pediatric population was recognized in 2002 (ref. 84), and a prevalence ranging from 17 to $60 \%$ was found across different age groups in patients with GSD Ia. The cause of anemia in GSD I is multifactorial-the restricted nature of the diet, chronic lactic acidosis, renal involvement, bleeding diathesis, chronic nature of the illness, suboptimal metabolic control, ${ }^{40}$ hepatic adenomas, ${ }^{29}$ and irritable bowel disease in GSD Ib are all contributing factors. In one study it was noted that patients with hemoglobin concentrations 2 SDs below the mean for their age had higher mean daily lactate concentrations as compared with the nonanemic population ( 3.2 vs. $1.9 \mathrm{mmol} / \mathrm{l}$ ). An association between severe anemia and large hepatic adenomas was identified as well. ${ }^{29}$

Many patients with GSD I have iron deficiency anemia. In some, it is an iron refractory anemia attributable to aberrant expression of hepcidin. ${ }^{29}$ Hepcidin is a small peptide hormone produced in the liver. It is secreted in the bloodstream and is the key regulator of iron in the body, controlling iron absorption across the enterocyte, as well as macrophage recycling of iron. In the presence of hepatic adenomas, there are increased hepcidin levels. The inability of hepcidin to be downregulated in the setting of anemia causes abnormal iron absorption and iron deficiency. Intravenous iron infusions can partially overcome the resistance to iron therapy, but, because of an inhibition of macrophage recycling of iron, a good response is typically not seen.

The restricted nature of the diet, with a focus on maintaining normoglycemia, often results in nutritional deficiencies (see Nutrition section) including poor intake of iron, vitamin B12, and folic acid. Progression of kidney disease is another risk factor for anemia, and some patients require supplementation with EPO to maintain hemoglobin levels.

\section{Anemia in type lb}

The causes of anemia in GSD Ib are similar to those of anemia in GSD Ia, as was noted in five subjects studied by Talente et al. ${ }^{114}$ in 1994. Numerous case reports documented the presence of anemia in this population, but studies of the pathophysiology of this complication were lacking. Interleukin 6-a marker of inflammation known to upregulate hepcidin expression, which is increased during inflammatory bowel disease exacerbations - is the likely cause of low hemoglobin concentrations and another cause for the anemia observed in patients with GSD Ib.

A larger study involving 202 subjects with GSD I at two large GSD centers has shed more light on the causes of anemia in GSD I. ${ }^{144}$ In this study it was noted that in the GSD Ia population, the prevalence of anemia increases with age. Mild anemia is common in the pediatric population because of iron deficiency and dietary restrictions. As previously stated, overall, pediatric patients with anemia have worse metabolic control, but the anemia is responsive to improved therapy and iron supplementation. By contrast, anemia in adulthood is associated with hepatic adenoma formation, particularly in people with more severe anemia. More than $85 \%$ of patients with hematocrit values less than $30 \%$ have large hepatic adenomas exceeding $5 \mathrm{~cm}$ in diameter. The finding that all subjects who had resection of the dominant hepatic adenoma experienced resolution of their anemia supports the proposed pathophysiology of hepcidininduced anemia.

Of the patients with GSD Ib $(n=39)$, anemia was present in $72 \%$. In contrast to the GSD Ia population, there was no association between anemia and metabolic control or hepatic adenomas in either children or adults with GSD Ib; however, a strong association with systemic inflammation was documented. Severe anemia was much more common in the patients with GSD Ib, and those with hematocrit values less than $30 \%$ were found to have active GSD enterocolitis. The enterocolitis in GSD Ib histologically resembles inflammatory bowel disease/ Crohn disease with transmural inflammatory changes and granuloma formation. ${ }^{100}$

\section{Bleeding diathesis}

In GSD I, a coagulation defect attributed to acquired platelet dysfunction with prolonged bleeding times, decreased platelet adhesiveness, and abnormal aggregation has been described (Box 5). Some patients with GSD I have features suggestive of a von Willebrand disease-like defect with reduced von Willebrand factor antigen and/or dysfunctional von Willebrand factor. Bleeding manifestations include epistaxis, easy bruising, menorrhagia, ${ }^{45}$ and excessive bleeding during surgical procedures. Although dietary intervention can ameliorate the 
bleeding diathesis, the exact etiology of the bleeding diathesis remains unclear. More than one study, with limited numbers of patients, showed that infusions of glucose and total parenteral nutrition corrected the bleeding time and in vitro platelet function in patients with GSD I, suggesting that coagulation defects were secondary to metabolic abnormalities. ${ }^{27,56,57}$

Standard management of patients with platelet dysfunction/ von Willebrand disease include antifibrinolytics and deamino-8-D-arginine vasopressin, which acts by stimulating factor VIII from endothelial cells and improving von Willebrand factor activity and the platelet release reaction. These agents could be utilized in patients with GSD I when clinically indicated, but use of deamino-8-D-arginine vasopressin in GSD I must be performed with caution because of the risk of fluid overload and hyponatremia in the setting of i.v. glucose administration..$^{145,146}$

In addition, the use of a fibrinolytic inhibitor, such as $\varepsilon$-aminocaproic acid (Amicar), can be used as an adjunctive medication if there is mucosal-associated bleeding. For oral hemorrhage, Amicar can be given as a solution to "swish for 30 seconds and spit" at a dose of $1.25 \mathrm{~g}$ four times daily. For more severe mucosal-associated bleeding, an i.v. bolus of $4 \mathrm{~g}$ in $250 \mathrm{ml}$ of D5W/NS infused over 1 hour followed by a drip of $1 \mathrm{~g} / \mathrm{h}(50 \mathrm{ml} / \mathrm{h})$ for 8 hours or until bleeding is controlled is needed. If the i.v. form is not available and the patient can take oral medications, $5 \mathrm{~g}$ may be given in the first hour, followed by $1 \mathrm{~g} / \mathrm{h}$ orally for $8 \mathrm{~h}$ or until hemorrhage is controlled. The use of Amicar is contraindicated in individuals with disseminated intravascular coagulation and if activated prothrombin complex concentrate (FEIBA) has been used. Caution must be taken to ensure that there is no genitourinary tract bleeding, because inhibition of fibrinolysis can lead to an obstructive nephropathy.

\section{Neutropenia, neutrophil dysfunction, and enterocolitis in GSD lb}

Neutropenia and recurrent infections are common manifestations of GSD Ib. In a European survey of 57 patients with GSD $\mathrm{Ib}, 49$ patients $(86 \%)$ had severe neutropenia with an absolute neutrophil count (ANC) less than $0.5 \times 10^{9} / 1$ (ref. 147). Neutropenia persists throughout childhood with little change in the neutrophil levels. ${ }^{147}$ However, some children with GSD Ib, who develop neutropenia later in life, have normal neutrophil counts early in life. It is unclear if neutrophil function is normal in this setting. Adult patients also have severe neutropenia and recurrent infections. ${ }^{148}$ Patients are prone to development of gingivitis, mouth ulcers, upper respiratory infections, deep abscesses, and enterocolitis. The patterns of infections vary from patient to patient, but there is no clear genotypephenotype relationship. ${ }^{114}$

Neutropenia and the susceptibility to infections are now attributed to specific abnormalities in neutrophil production and function. ${ }^{149}$ Glucose is critical for the neutrophil's metabolic burst, which occurs with the ingestion of microorganisms. Mutations in glucose 6-phosphate transporter (G6PT) cause apoptosis of developing neutrophils, ineffective neutrophil production, and neutropenia. ${ }^{150}$ The mature cells that survive to enter the blood have a reduced metabolic burst when they ingest particles or microorganisms. Monocyte functions are also abnormal, probably contributing to the formation of granulomas and chronic inflammatory responses. ${ }^{151}$

It is also important to note that some patients with GSD Ia have also been known to develop neutropenia. Individuals with GSD Ia who are homozygous for the mutation p.Gly188Arg were reported to have a GSD Ib-like phenotype with neutropenia. ${ }^{6}$

\section{Neutropenia treatment}

G-CSF has been used for treating neutropenia and preventing infections in patients with GSD Ib since 1989 (refs. 152,153). This cytokine stimulates and accelerates neutrophil production by the bone marrow, releases neutrophils from the bone marrow, prolongs the survival of the cells, and enhances their metabolic burst. Administration of G-CSF increases blood neutrophil counts to normal or above normal levels, usually within a few hours. In a review of 18 European patients given either glycosylated or nonglycosylated G-CSF (median age: 8 years; treated for up to 7 years), there was a positive clinical response both in the severity of infections and in the manifestations of inflammatory bowel disease in all patients. ${ }^{147}$ One patient had relapses in bowel disease while taking G-CSF, but the others did not. Almost all reports on GSD Ib indicate that G-CSF increases blood neutrophil levels, decreases the occurrence of fevers and infections, and improves enterocolitis. ${ }^{154,155}$ The cytokine granulocyte macrophage colony-stimulating factor has also been used on a short-term basis, but it is not as well tolerated on a long-term basis as G-CSF. The Severe Chronic Neutropenia International Registry (a National Institutes of Health/National Institute of Allergy and Infectious Diseasessponsored registry based at the University of Washington, Seattle, WA) maintains records for 37 patients with GSD Ib (23 adults ( 16 female, 7 male) and 14 children (9 female, 5 male); age 4.1-39.5 years; DC Dale et al., unpublished data). Before G-CSF treatment, median ANC for this group was $0.44 \times 10^{9} / 1$. Treatment can be performed daily, on alternate days, or on a Monday-Wednesday-Friday schedule with similar benefits (DC Dale, personal communication), but some children require daily therapy to avoid infections. In the United States, the dose used is generally low, approximately $2 \mu \mathrm{g} / \mathrm{kg} /$ day. G-CSF should be administered subcutaneously starting at $1.0 \mu \mathrm{g} / \mathrm{kg} /$ day given daily or every other day. The G-CSF dose should be increased in a stepwise manner at approximately 2 -week intervals until the target ANC of more than 500 to up to $1.0 \times 10^{9} / 1$ is reached. The ANC for these patients is not pushed to higher levels because G-CSF appears to increase the spleen size in GSD Ib patients. This dose then should be maintained, adjusting for subsequent increases in the patient's weight with growth and development. Blood count should be monitored several times per year. Bone marrow examinations are not recommended, unless there is an unexpected change in the patient's other blood counts. The 
lowest effective G-CSF dose should be used to avoid splenomegaly, hypersplenism, hepatomegaly, and bone pain.

With use of G-CSF, occurrences of infections were greatly reduced and inflammatory bowel disease also improved in most, but not all, patients. In more than 470 patient-years of observations, the Severe Chronic Neutropenia International Registry has recorded three deaths in GSD Ib patients, (sepsis, 1; after liver and hematopoietic transplant, 1; hepatomegaly and neutropenia, 1).

Side effects of treatment with G-CSF in the GSD Ib population were reported by the European Study on Glycogen Storage Disease Type I. ${ }^{147}$ The most serious complication was splenomegaly. This complication did regress with reduced treatment. There are known cases in which the splenomegaly did not improve with reduction of the dose and splenectomy was required. Increase in spleen size and the need to reduce G-CSF dose can usually be determined by physical examination and confirmed by ultrasound when necessary. In addition, this group reported two patients that have been on G-CSF and developed acute myelogenous leukemia. Based on available data, the risk of acute myelogenous leukemia is very low. However, all patients should be observed, with serial blood

\section{Box 5 Hematology recommendations}

- Evaluation for anemia should include nutritional causes, adenomas, enterocolitis, menorrhagia in females, and occult blood loss. Evaluations should include complete blood cell count with manual differential, serum, total iron-binding capacity, and reticulocyte counts.

- In GSD la, if anemia is severe, evaluation for hepatic adenomas should be performed.

- In GSD Ib, if anemia is severe, evaluation for GSD enterocolitis should be performed.

- If iron deficiency anemia is documented, iron supplementation (oral or i.v.) as needed and optimization of metabolic control are recommended. Consider iron refractory anemia if iron levels do not improve.

- Neutropenic patients with GSD Ib should be treated with G-CSF, particularly if there is already a history and pattern of fever, infections, or enterocolitis.

- The lowest effective G-CSF dose should be used to avoid worsening of splenomegaly, hypersplenism, hepatomegaly, and bone pain. G-CSF should be administered subcutaneously starting at $0.5-1.0 \mu \mathrm{g}$ per kilogram per day given daily or every other day. The G-CSF dose should be increased stepwise at approximately 2-week intervals until the target ANC of more than 500 to up to $1.0 \times 10^{9} / \mathrm{l}$ is reached. This dose then should be maintained, adjusting for subsequent increases in the patient's weight with growth and development.

- Blood count with manual differential should be monitored several times per year. Bone marrow examinations are not recommended unless there is an unexpected change in the patient's other blood counts. counts monitored approximately quarterly for development of loss of response to G-CSF, presence of myeloblasts in the blood, evidence of hypersplenism, new patterns of bone pain, or any other changes that might suggest a change in hematological disease or development of a myeloid malignancy.

\section{CARDIOVASCULAR ASPECTS}

In contrast to the hypertrophic cardiomyopathy of GSD II (Pompe disease) or GSD III, the heart itself is not primarily affected by GSD I. The most common cardiovascular abnormality in patients with GSD I is systemic hypertension (Box 6). ${ }^{84}$ It usually occurs in the context of renal disease and does not develop until the second decade of life or later, with the median age of onset 17 years. ${ }^{84}$ The diagnosis, monitoring, and treatment of systemic hypertension is similar to that of essential hypertension in children. This is reviewed in the Nephrology section of this article. Hypertension and hyperlipidemia are both independent risk factors for the development of atherosclerosis later in life; thus, it is pertinent to question whether individuals with GSD I are at increased risk for and/or earlier risk for development of atherosclerosis as adults. There are conflicting data about this question, and two small series examining clinical surrogates of early atherosclerosis found no evidence to suggest early atherosclerosis. ${ }^{119,156}$ Nonetheless, there are a few case reports of patients with early coronary artery disease. ${ }^{114} \mathrm{It}$ is likely that early atherosclerosis is a rare complication of GSD I and management should aim to maintain normal blood pressure, renal function, and lipid levels to prevent its occurrence.

One of the most ominous, yet rare, potential complications of GSD I is the occurrence of pulmonary arterial hypertension (PAH). PAH may coexist with numerous systemic illnesses such as rheumatologic diseases, portal hypertension, infections (such as HIV), and exposure to toxins (anorexigens). PAH is also known to be a complication of several other conditions, such as hypoxic lung disease, thromboembolic disease, pulmonary venous hypertension secondary to left-sided heart disease, and congenital heart disease with left-to-right shunting through the lungs. Finally, it may occur in isolation as primary PAH. To date, nine GSD I patients with PAH have been reported. ${ }^{49,50,157-163}$ Of these, five had concomitant conditions that were also associated with the development of $\mathrm{PAH}$, including three patients with portocaval shunts, one patient with an atrial septal defect, and one patient with hereditary hemorrhagic telangiectasia. ${ }^{33,49,84,157-159,161}$ It has been suggested that abnormal handling of serotonin might be one event in a multistep process leading to PAH in GSD I patients. This suggests that the GSD I patient with a coexisting condition that may also predispose a patient to development of $\mathrm{PAH}$ is at the highest risk for this complication. In all the cases of GSD I with PAH described in the literature, the diagnosis of PAH was not made until it was quite advanced, and in seven of nine patients PAH led to their deaths. ${ }^{33,49,84,157-161}$ There is one recent case report of a patient with significant $\mathrm{PAH}$ with systemic rightventricular pressure who responded well to medical management with prostacyclin and sildenafil (Viagra) to reduce the pulmonary artery pressure. ${ }^{162}$ Historically, there have been few effective 
long-term treatment modalities available for PAH. Recently, oral medications for PAH, such as sildenafil, have been shown to be effective treatments. GSD I patients with this serious complication have a better chance of longer survival if $\mathrm{PAH}$ is diagnosed at an earlier stage and medical treatment is initiated promptly.

Management recommendations for cardiovascular manifestations of GSD I include screening to detect systemic or pulmonary hypertension at early stages when these conditions are most amenable to treatment. Because systemic hypertension in children is only rarely associated with clinical symptoms such as headaches or vision changes beginning in infancy, accurate measurements of systemic blood pressure should be obtained at all clinic visits. Any elevated blood pressure measurements should be carefully followed up to confirm the diagnosis of hypertension. It is important to note that age-appropriate and gender-appropriate norms for blood pressure should be applied when reporting it.

Good metabolic control is the best management option for maintaining serum lipid levels as close to normal as possible, thereby reducing the risk of acute pancreatitis and long-term development of atherosclerosis. Management of hyperlipidemia with medications usually does not begin until the patient is at least 10 years old.

Screening for pulmonary hypertension by periodic echocardiography with attention to estimating right-ventricular pressure by tricuspid regurgitation jet is indicated because PAH is unlikely to have clinical features that would be apparent on physical examination or with simple testing such as electrocardiogram until the PAH is well advanced. Obtaining the tricuspid regurgitation jet by echocardiogram is the best method to periodically screen for elevated right-side heart pressures. ${ }^{163}$ Because the reported cases of GSD I patients with PAH were mostly children older than 10 years of age, screening by echocardiography can likely be delayed until patients are 10 years of age or older. ${ }^{33,84}$ Periodic screening echocardiograms could be performed every 3 years or at shorter intervals if there are any suggestive clinical symptoms. Because most of the patients with PAH also had poor metabolic control, achieving good metabolic control may prevent PAH. If PAH is detected, pursuing effective treatment methods such as treatment with Bosentan and Sildenafil in consultation with a physician experienced in managing $\mathrm{PAH}$ is recommended.

\section{Box 6 Cardiovascular recommendations}

- Screen systemic blood pressure to detect systemic hypertension beginning in infancy.

- Maintain lipid levels within the normal range to prevent atherosclerosis and pancreatitis.

- Screen for pulmonary hypertension by periodic echocardiography with attention to estimating right-ventricular pressure by tricuspid regurgitation jet starting at age 10 years and repeating every 3 years or at shorter intervals if there are suggestive clinical symptoms.

\section{GENERAL MEDICAL CARE}

All patients with GSD should have a primary-care provider ("medical home") specializing in pediatrics, adolescent, or internal medicine depending on the patient's age (Box 7). The primary-care physician should take care of the regular physical examinations and immunizations, as well as any intercurrent medical problem not related to the GSD. He/she should be familiar with the major manifestations of GSD I and should maintain good communication with the patient's specialists as needed. Some patients/families find it useful to have a binder in which they can keep physician cards, insurance information, authorizations, school evaluations, and/or other important documents.

Routine immunizations should be scheduled as recommended by the Centers for Disease Control and Prevention (http://www.cdc.gov/vaccines/schedules/). Other available immunizations, such as those for seasonal influenza, hepatitis $\mathrm{B}$, and pneumococcal infections (polyvalent after 2 years of age), should be offered because they can prevent the hypoglycemia caused by the gastrointestinal manifestations associated with the disease processes. Hepatitis $\mathrm{C}$ status should be monitored in patients at risk.

Because patients with GSD I may receive several medications, it is always recommended to check for potential interactions with the physician or pharmacy when a new medication is prescribed. Drugs that can potentially cause hypoglycemia should be avoided. These include $\beta$-blockers, quinidine, sulfonamides (Bactrim), pentamidine, and haloperidol, as well as some overthe-counter medications. Antidepressant agents should be used with caution because they can affect glucose regulation (hypoglycemia or hyperglycemia). Insulin and insulin secretagogues (sulfonylureas) should be used with caution. ${ }^{164,165}$ Growth hormone treatment should also be avoided because it can result in the development or an increase in the size or number of liver adenomas, along with severe hyperlipidemia. The use of growth hormone should clearly be limited to only those who are proven to have a growth hormone deficiency and, in this situation, close monitoring for liver adenomas and metabolic disturbances is critical. The use of aspirin, nonsteroidal antiinflammatory drugs, and other medications that reduce or affect platelet function should be avoided. Hypoglycemia risks should be checked before starting medications.

Due consideration should be given to medications that have a high sodium or potassium content; the latter is especially important in the setting of renal failure.

All patients should be encouraged to participate in ageappropriate physical activities. However, contact or competitive sports should be avoided because of the risk of liver injury, unless proper protection is used. Patients should avoid alcohol intake as it may predispose them to hypoglycemia. Good hygiene and frequent hand-washing precautions are advised, especially for patients with neutropenia. As a general rule, patients should avoid unnecessary contact with sick people, especially during the winter season. Good dental hygiene and frequent monitoring of dental health are advised for all patients, but it is particularly important in patients with GSD Ib, who have a tendency 
to develop chronic gingivitis. Antibiotic coverage is not needed for routine dental cleaning, but it is indicated in the case of gingivitis or in case of oral infections with risk for cellulitis/sepsis due to neutropenia.

During intercurrent illnesses, early evaluation and treatment are encouraged to prevent complications, especially when infectious processes are suspected in patients with neutropenia. When the intercurrent illness causes decreased dietary intake, the patient's specialist should be contacted. In such cases more frequent monitoring of BG and additional doses of CS may be indicated. Patients who cannot maintain normal dietary intake/CS treatment or who have emesis should proceed to the nearest emergency department for evaluation and i.v. glucose treatment. The patient's specialist should be made aware and, ideally, should contact the emergency department in advance to ensure that patient's waiting time in the emergency department is minimized. Some emergency departments have implemented a "pathway" system for metabolic patients to reduce the waiting time until the proper care is started. ${ }^{166}$

All patients/families should be provided with an emergency letter that should be carried at all times. Patients/families should have several copies of this letter and keep them, for example, in their wallet/purse, car glove compartment, school file, and workplace. If possible, the emergency letter should be uploaded into the patient's electronic medical records for easy access. The emergency letter should be reviewed annually and updated as needed.

Patients should wear a medical alert identification. A variety of types are offered by pharmacies and websites:

- Necklaces and bracelets with engraved patient name, diagnosis, and emergency contact information.

- MedicAlert system (http://www.medicalert.org) offers a sponsored membership program that provides bracelets with an engraved toll-free telephone number and patient ID number. By calling the toll-free number, the patient's vital information, emergency contact, and other information can be obtained at any time, from anywhere.

- Electronic medical alert bracelets have a built-in "USB pen-drive" containing key medical information that can be accessed by plugging it into any computer with a USB port.

- An ICE (in case of emergency) telephone number should be listed in the patient's cell phone.

Patients are advised to carry an emergency kit at all times. The kit should include:

- Contact information;

- A copy of the emergency letter;

- Glucose meter, strips, extra battery, flashlight;

- Water;

- CS, glucose gel; and

- Cereal bar or similar source of carbohydrate.

\section{Box 7 General medical care recommendations}

- All patients should have a primary-care provider ("medical home").

- Routine immunizations should be given as recommended by the Centers for Disease Control and Prevention.

- Avoid medications that can potentially cause hypoglycemia and check for potential drug interactions/side effects when a new medication is prescribed.

- Patients should be encouraged to participate in age-appropriate physical activities, but contact or competitive sports should be avoided because of the risk of liver injury.

- Good dental hygiene and frequent monitoring of dental health are advised.

- During intercurrent illnesses, early evaluation and treatment are encouraged. Patients who cannot maintain normal dietary intake/CS treatment or who have emesis should proceed to the nearest emergency department for evaluation and i.v. glucose treatment.

- All patients/families should carry an emergency letter and an emergency kit at all times (see sample).

- All patients should wear a medical alert identification.

\section{SURGERYIANESTHESIA}

Metabolic derangement caused by fasting and infections are a common cause of morbidity in patients with GSD I, even with current treatments. ${ }^{84}$ In the case of surgical procedure, the metabolic center should be contacted in advance to provide recommendations for management (Box 8).

During infections or other intercurrent illnesses, the patient's glucose requirement may increase. In addition, some illnesses causing anorexia and vomiting interrupt oral or nasogastric feedings. Patients and their parents should be educated regarding the symptoms of hypoglycemia and metabolic decompensation. They should be taught to respond to minor ailments by giving frequent oral or NG glucose-containing fluids, and they should be educated regarding the need for emergency care if oral feeds are not tolerated.

In the case of loss of consciousness or inability to tolerate oral or NG feedings, glucose infusion rates by age have been published and need to be individualized based on the patient's needs. ${ }^{84}$ In emergency settings, i.v. glucose-containing fluids consisting of dextrose $10 \%$ with electrolytes administered at $1.5-2 \times$ the usual maintenance rate will generally increase BG levels to the normal range and reverse catabolism. Of course, due consideration of fluid volume is given in the setting of renal failure. Intravenous solutions containing lactate are contraindicated and should be avoided.

Careful management of the patient's glucose and electrolyte levels around the time of surgical procedures is needed. Patients with GSD I cannot tolerate typical periods of fasting before procedures. Progressive metabolic acidosis and cardiac dysrhythmia leading to cardiac arrest during surgery have been 
reported. Recommendations have been published as a guide for perioperative management. ${ }^{167,168}$ The patient should be admitted to the hospital on the day before the procedure so that a continuous i.v. supply of glucose can be provided. The recommended starting rate of $10 \%$ dextrose in 0.5 normal saline (or age-appropriate electrolytes) is $1.25-1.5 \times$ the maintenance rate. The i.v. fluid rate should be adjusted to keep the BG higher than $70 \mathrm{mg} / \mathrm{dl}$. BG, electrolyte, and lactic acid levels should be monitored. Intraoperatively, 10\% dextrose solutions should be continued with intraoperative monitoring of BG and lactic acid levels. Although administration of dextrose-containing fluids at lower rates can result in normalization of BG, higher doses of glucose are needed to keep the patient anabolic and prevent lactic acidosis. ${ }^{169}$ The i.v. fluids should continue until oral feeding is re-established. Lactated Ringer's should not be used as an i.v. fluid. Once the patient is taking oral feedings, the dextrose infusion should be slowly weaned over several hours.

Prolonged bleeding after surgery was reported in 3\% of patients with GSD I in the European Study on Glycogen Storage Disease. ${ }^{33,84}$ Complications attributable to bleeding tendency were reported in $23 \%$ of patients, with most being due to recurrent or severe epistaxis. ${ }^{33}$ The exact mechanism of the bleeding tendency is unknown; however, defects in platelet aggregation have been found and the bleeding time is prolonged (see section on Hematology). It has been recommended that bleeding time be normalized by continuous gastric drip feedings for $24 \mathrm{~h}$ for 1 week before surgery; however, a more practical solution may be continuous i.v. glucose for $24 \mathrm{~h}$ before surgery. ${ }^{33,84}$

\section{GYNECOLOGICAL/OBSTETRICAL CARE}

Caution should be used when prescribing hormonal birth control; estrogen is known to contribute to development of both benign and malignant hepatocellular tumors (Box 9). ${ }^{170}$ Given the risk of adenomas in GSD I patients, estrogen-based contraceptives should be avoided when possible. ${ }^{107}$ Progestinonly contraceptives do not pose the same risk and may be

Box 8 Surgery/anesthesia recommendations

- Educate parents about symptoms of hypoglycemia and metabolic decompensation.

- Enable parents to provide oral or NG glucose during minor ailments.

- Parents should be provided an emergency treatment plan.

- Careful management of the patient's glucose and electrolytes during surgery is necessary.

- Admission $24 \mathrm{~h}$ before a surgical procedure allows for i.v. fluids with D10 and metabolic control.

- Lactated Ringer's solution should not be used because of the risk of worsening lactic acidosis and metabolic decompensation.

- Intravenous glucose-containing fluids or nutrition (total parenteral nutrition when indicated) should not be discontinued abruptly; this should be performed only after the patient is eating and maintaining blood glucose levels. considered; however, there are other challenges such as risk for reduced bone mineral density, which needs to be monitored. ${ }^{171}$ In GSD Ib, use of an intrauterine device should be avoided. Females with GSD I are known to have polycystic ovaries from a young age. ${ }^{46}$ Fertility is not thought to be reduced.

Menorrhagia appears to be a problem in females of reproductive age with GSD I. ${ }^{44,45}$ Interventions have included hormonal and nonhormonal treatments. Specific questions documenting the occurrence of menorrhagia or irregular menstrual bleeding should be part of the patient's history; this area seems to be often overlooked because care providers are focused on other aspects of the disease. Management of females with GSD I should include a multidisciplinary approach including the expertise of a gynecologist familiar with GSD I. ${ }^{45}$ This complication should be considered when reviewing the history of female patients with GSD I and in the management of these cases.

\section{Pregnancy}

With significant strides in management of GSD I, patients are surviving into adulthood and pregnancies are now becoming common. Successful pregnancies have been documented in women with GSD types Ia and Ib. . $^{47,48,105,106,108}$

Ideally, it is prudent to plan the pregnancy ahead of time so that metabolic parameters may be monitored and normalized in preparation for pregnancy. A prepregnancy consultation should be conducted during which adherence to a safe diet routine to avoid low BG, accompanied by frequent BG monitoring, should be emphasized. Medications such as ACE inhibitors, allopurinol, and lipid-lowering drugs must be discontinued because they are known to cause fetal anomalies. A baseline ultrasound of the kidneys and liver to monitor for hepatic adenomas should be performed before the patient becomes pregnant. Laboratory tests such as a lipid profile, serum uric acid test, liver function test, complete blood count, and urine protein test should be performed. Good metabolic control will help normalize most of these parameters if abnormal. In addition, in patients with GSD $\mathrm{Ib}$, conception at a time when inflammatory bowel disease is quiescent may make flare-ups during pregnancy less likely. ${ }^{172}$ The patient may benefit from a referral to a high-risk obstetrician because care during pregnancy will require multidisciplinary involvement.

Abdominal ultrasound; urine microalbumin/creatinine, serum uric acid, cholesterol, triglyceride-level, and liver enzyme tests; and complete blood counts should be performed every 3 months. The high estrogenic state in pregnancy has been reported to cause an increase in adenoma formation. ${ }^{173}$ Worsening of renal function can occur, especially if it is already compromised. Increased proteinuria may be noted. Risk of stone formation is typically higher in GSD Ia than in GSD Ib, ${ }^{40}$ but renal calcification was noted in two of three pregnant patients with GSD Ib in one case series. ${ }^{108}$ Serum uric acid and serum triglycerides levels should be monitored because elevations can occur when a patient has poor metabolic control. 
Neutropenia and Crohn disease-like enterocolitis are problems unique to GSD Ib. Low neutrophil counts can lead to infectious complications. G-CSF is classified by the US Food and Drug Administration as a pregnancy class $\mathrm{C}$ drug. There are no recommendations for G-CSF use during pregnancy. There are published reports in the literature of normal pregnancy outcomes after G-CSF use. ${ }^{174-180}$ The decision to start or continue G-CSF should be made in consultation with multiple specialists weighing the risks versus the benefits. Management of Crohn diseaselike enterocolitis can be problematic in pregnancy because most medications used for treatment are not approved for use during pregnancy. The risk to the fetus from active enterocolitis has to be considered in comparison with the risk from the medications themselves during decision making regarding management.

In preparation for labor and delivery, a protocol with written instructions must be available stressing the use of $10 \%$ dextrose solution given intravenously during labor and delivery to prevent hypoglycemia. Once the patient is in labor, close collaboration between the obstetrician and the patient's specialist is imperative. BG levels should be monitored throughout the process to maintain euglycemia. Transient hypoglycemia has been observed in some neonates. Neonates have been noted to have normal growth and development. There is no contraindication to breastfeeding. Increased metabolic demands will occur while breastfeeding. It has been observed that not all mothers may be successful at breastfeeding.

\section{CARE COORDINATION AND SUPPORT}

The Association of Glycogen Storage Disease US (http://www. agsdus.org/) is an organization that provides information and support to people with GSD and their families. The website provides descriptions of the various types of GSD and a listserv, a mechanism for people with all forms of GSD to connect via the Internet. The association also holds a medical conference each year for individuals with GSD and their families.

\section{Box 9 Gynecological/obstetrical recommendations}

- Avoidance of estrogen as an oral contraceptive, because of increased risk for adenoma formation, is recommended. For GSD $\mathrm{Ib}$, avoidance of an IUD because of increased infection risk is recommended.

o Progestin-only contraceptives may be considered. There is a risk for reduced bone mineral density, which needs to be monitored.

- Evaluate for menorrhagia and refer as appropriate.

- Plan pregnancy so that metabolic parameters may be monitored and normalized in preparation for pregnancy.

- Medications such as ACE inhibitors, allopurinol, and lipid-lowering drugs must be discontinued during pregnancy.

- BG levels and overall metabolic control (including renal status) should be monitored during pregnancy and labor to maintain euglycemia.

- Pregnancies should be followed by a high-risk $O B$ in a tertiary setting.

\section{GENETIC COUNSELING, PRENATAL DIAGNOSIS, AND SCREENING}

Similar to that for other inborn errors of metabolism, genetic counseling should be offered to all parents of children with GSD I and to adults affected with the condition (Box 10). In counseling families with GSD I, at least a three-generation pedigree from the consult and/or proband should be obtained. GSD I is an autosomal recessive condition. De novo mutation rates are expected to be infrequent, and parents of an affected individual are assumed to be carriers. The recurrence risk for parents who have had an affected child is $25 \%$. DNA mutation analysis is necessary for the identification of additional family members in the extended family who may be carriers. Several laboratories in the United States offer DNA diagnostic and/or prenatal diagnostic testing for GSD I (see http://www.genetests.org). Targeted mutation analysis based on ethnic background is available for both the G6PC and SLC37A4 genes. Generally, full sequence analysis is recommended, starting with GSD Ia and then GSD Ib, if clinical suspicion is present. Sequence analysis detects $\sim 94 \%$ of mutations in mixed populations. Large deletions and duplications cannot be detected by sequence analysis. Identification of carrier status in the general population is limited and not routinely offered; however, mutation analysis to further refine the risk of having a child with GSD I can be offered to those at risk (e.g., the spouse of a known carrier or spouse of an affected person).

Prenatal diagnostic testing is typically performed by mutation analysis either on cultured chorionic villus samples or on amniocytes, ideally of the probands of previously identified mutations. When the mutations segregating in the family are known, molecular testing is the gold standard. Prenatal genetic diagnosis is also an option for families with GSD I if the mutations have been identified.

\section{Box 10 Genetic counseling/prenatal diagnosis/screening} recommendations

- Offer genetic counseling to all parents with a child with GSD I and to all adults with GSD I.

- Determine the proband's G6PC / SLC37A4 mutations when feasible for diagnosis and to direct further testing for family members.

- When both mutations are known, molecular testing is the preferred method for prenatal diagnosis.

\section{FUTURE DIRECTIONS AND EMERGING THERAPIES}

Acute and chronic complications occur in GSD Ia despite adherence to dietary therapy, including growth retardation, hepatomegaly, intermittent hypoglycemia, lactic acidemia, hyperlipidemia, gout related to hyperuricemia, proteinuria, nephrolithiasis, and progressive nephropathy. Modified CS shows promise for improving dietary therapy because a single dose at bedtime prevented hypoglycemia more effectively throughout the night in comparison with uncooked CS. ${ }^{86}$ 
Perhaps one of the most concerning complications of GSD I is the frequent occurrence of hepatic adenomas in adult patients, which are accompanied by a significant risk for malignant transformation to HCC. ${ }^{120,121}$ Chromosome 6 abnormalities have been demonstrated in hepatic adenomas from GSD Ia patients. ${ }^{120,121}$ Expression of two candidate tumor suppressor genes at $6 \mathrm{q}$ was reduced in more than $50 \%$ of these hepatic adenomas, indicating that loss of these genes might reflect an early event in tumorigenesis in the liver. The mechanism for tumorigenesis remains to be elucidated in GSD Ia, although it could include chronic inflammation. Intriguingly, a subclinical abnormality of neutrophil metabolism was reported in G6Pase $(-/-)$ mice ${ }^{181}$; furthermore, neutrophil infiltrates were demonstrated in human GSD Ia livers accompanied by elevated interleukin 8, consistent with ongoing inflammation. ${ }^{182}$

Progressive nephropathy is associated with proteinuria in adult patients. ${ }^{188}$ It has been postulated that excessive reninangiotensin activity could underlie hyperfiltration and progressive renal failure in GSD Ia. ${ }^{133}$ To test this hypothesis, angiotensinogen was quantified and found to be elevated in kidneys of 2-week-old G6Pase(-/-) mice. Subsequently, increases in transforming growth factor- $\beta$ and connective tissue growth factor were demonstrated in older G6Pase(-I-) mice, in association with increases by angiotensinogen expression. ${ }^{184}$ Thus, the nephropathy of GSD Ia was associated with angiotensinogen overexpression in G6Pase(-/-) mice, even without associated proteinuria. ${ }^{184}$ The renin-angiotensin system plays a key role in renal failure in GSD Ia and needs further study.

The overexpression of angiotensinogen suggests that suppression of the renin-angiotensin system might be effective in GSD Ia. Microalbuminuria has been effectively treated with low doses of ACE inhibitors such as captopril and lisinopril. ${ }^{142}$ At low doses, these medications improve renal perfusion. In a study of 95 patients with GSD I, a significant and progressive decrease of glomerular hyperfiltration was noted in patients treated with ACE inhibitors. ${ }^{142}$ These medications also slowed the progression from hyperfiltration to microalbuminuria; however, ACE inhibitors did not slow progression from microalbuminuria to frank proteinuria and renal failure. ${ }^{142}$

Hyperlipidemia in GSD Ia can be managed with lipid-lowering drugs such as 3-hydroxy-3-methyl-glutaryl-CoA reductase inhibitors and fibrates. The potential benefit of 3-hydroxy3-methyl-glutaryl-CoA reductase inhibitors was emphasized by a study that showed increased triglyceride synthesis in GSD Ia patients compared with normal controls. ${ }^{185}$ Lowering triglycerides could reduce the risk for pancreatitis in patients with poor metabolic control, but no consensus regarding the recommendation of lipid-lowering drugs has been reached.

Hyperuricemia in GSD I can improve with good metabolic control; however, in some situations, hyperuricemia persists and can result in gouty attacks, gouty tophi, and kidney stones. Use of agents, such as Allopurinol and Febuxostat, have been used to lower uric acid levels. Newer agents, such as pegloticase, have been used in situations where the use of other agents has failed. Colchicine has been used with success in the acute setting of gouty attacks. At this time, there is no consensus as when to treat hyperuricemia with medications.

The development of new therapy for GSD Ia, such as gene therapy or cell therapy, might prevent long-term complications that arise due to recurrent hypoglycemia and related biochemical abnormalities. Pilot studies of hepatocyte transplantation have demonstrated persistence of donor cells, although the long-term efficacy of this approach remains to be demonstrated ${ }^{186,187}$ Efficacy from liver-targeted gene therapy in GSD Ia might be expected, given the experience with human patients after liver transplantation. Adeno-associated virus (AAV) vectors containing a human G6Pase regulatory cassette/promoter have proven to be efficacious in animal models of GSD Ia, and these vectors contain sequence elements that regulate G6Pase expression appropriately. ${ }^{188} \mathrm{~A}$ small-genome, double-stranded AAV2/8 vector containing a human G6Pase minigene demonstrated high efficacy in G6pase(-/-) mice and dogs with GSD Ia. ${ }^{183} \mathrm{~A}$ single-stranded AAV vector containing a larger human G6Pase regulatory cassette also showed high efficacy in G6pase (-/-) mice. ${ }^{189}$ Other vectors that have been evaluated in the G6pase (-/-) mouse model have included helper-dependent adenovirus vector encoding canine G6Pase ${ }^{183}$ and a feline immunodeficiency virus vector encoding murine G6Pase. ${ }^{190}$ Although both vectors prolonged survival and prevented hypoglycemia in the majority of treated mice, each remains limited by significant concerns related to potential toxicity, and more preclinical experiments will be needed to further evaluate the potential of these systems for clinical translation. Furthermore, complications of GSD Ib were incompletely reversed in experiments with an AAV vector encoding G6PT, and longer-term surviving mice developed hepatocellular carcinoma related to inadequate correction. ${ }^{191}$

The duration of efficacy from AAV vectors has been limited, because the AAV vector genomes remain largely episomal and are lost after cell division. A double-stranded AAV vector transduced the liver and kidneys with higher efficiency when pseudotyped as AAV9 rather than the AAV8 vector used for initial experiments; however, G6Pase expression from these vectors gradually waned between 7 and 12 months of age. ${ }^{192}$ Another study with a single-stranded AAV vector containing a larger G6Pase transgene completely corrected G6Pase deficiency in the liver at 6 months, followed by $90 \%$ loss of expression by 18 months of age in G6pase (-/-) mice. ${ }^{193}$ These studies show that although AAV vectors confer significantly longer expression than other common episomal gene therapy vectors (e.g., adenovirus or plasmid DNA vectors) ${ }^{194,195}$ expression from an $A A V$ vector does decrease over time. The loss of G6Pase could be countered by readministration of an AAV vector of a new serotype to avoid antibodies formed in response to the initial AAV vector treatment. ${ }^{196,197}$ In addition, G6pase(-/-) mice had decreased GH signaling, resulting in low Igf- 1 and slow growth, which was partially reversed by AAV vector administration. ${ }^{198}$ Therefore, concerns regarding safety and efficacy of gene therapy in GSD I remain to be addressed by additional long-term preclinical experiments. These experiments will be 
facilitated by newer animal models, including liver-specific G6pase(-/-) mice that develop all the liver involvement of GSD Ia, including adenomas and hepatocellular carcinoma when fed a high-fat diet. ${ }^{199}$ Liver-specific G6pase(-/-) mice that lack G6Pase only in the liver survive much better than complete G6pase(-/-) mice, which will facilitate long-term experiments to evaluate new AAV vectors in GSD Ia. Despite these apparent limitations of gene therapy in GSD I, the development of AAV vector-mediated gene therapy will continue based on the success of early-stage clinical trials of gene therapy in hemophilia. ${ }^{200,201}$

\section{ACKNOWLEDGMENTS}

The authors thank Areeg El-Gharbawy, Philippe Labrune, Yuan-Tsong Chen, and Kathy Ross for their critical comments and suggestions. They also thank Jennifer Goldstein for her help with editing this guideline and with correcting molecular mutation data. Partial funding of this guideline was provided by an educational grant from the Association of Glycogen Storage Disease that was restricted to use in developing practice guidelines for glycogen storage diseases. This study was approved by the American College of Medical Genetics and Genomics Board of Directors on 25 March 2014.

\section{DISCLOSURE}

The authors declare no conflict of interest.

\section{REFERENCES}

1. Gierke EV. Hepato-nephro-megalia-glycogenica (Glykogenspeicherkrankheit der Leber und Nieren). Beitr Pathol Anat 1929;82:497-513.

2. Cori GT, Cori CF. Glucose-6-phosphatase of the liver in glycogen storage disease. J Biol Chem 1952;199:661-667.

3. Narisawa K, Igarashi Y, Otomo H, Tada K. A new variant of glycogen storage disease type I probably due to a defect in the glucose-6-phosphate transport system. Biochem Biophys Res Commun 1978;83:1360-1364.

4. Lei KJ, Shelly LL, Pan CJ, Sidbury JB, Chou JY. Mutations in the glucose6-phosphatase gene that cause glycogen storage disease type 1a. Science 1993;262:580-583.

5. Lei KJ, Chen H, Pan CJ, et al. Glucose-6-phosphatase dependent substrate transport in the glycogen storage disease type-1a mouse. Nat Genet 1996;13:203-209.

6. Weston BW, Lin JL, Muenzer J, et al. Glucose-6-phosphatase mutation G188R confers an atypical glycogen storage disease type $1 \mathrm{~b}$ phenotype. Pediatr Res 2000:48:329-334.

7. Chou JY, Matern D, Mansfield BC, Chen YT. Type I glycogen storage diseases: disorders of the glucose-6-phosphatase complex. Curr Mol Med 2002;2: $121-143$

8. Ekstein J, Rubin BY, Anderson SL, et al. Mutation frequencies for glycogen storage disease la in the Ashkenazi Jewish population. Am J Med Genet A 2004;129A:162-164.

9. Janecke AR, Mayatepek E, Utermann G. Molecular genetics of type 1 glycogen storage disease. Mol Genet Metab 2001;73:117-125.

10. Kajihara S, Matsuhashi S, Yamamoto K, et al. Exon redefinition by a point mutation within exon 5 of the glucose-6-phosphatase gene is the major cause of glycogen storage disease type 1a in Japan. Am J Hum Genet 1995;57:549-555.

11. Lam CW, But WM, Shek CC, et al. Glucose-6-phosphatase gene (727G->T) splicing mutation is prevalent in Hong Kong Chinese patients with glycogen storage disease type 1a. Clin Genet 1998;53:184-190.

12. Matern D, Seydewitz HH, Bali D, Lang C, Chen YT. Glycogen storage disease type I: diagnosis and phenotype/genotype correlation. Eur J Pediatr 2002;161(suppl 1):S10-S19.

13. Rake JP, ten Berge AM, Verlind E, et al. Glycogen storage disease type la: four novel mutations (175delGG, R170X, G266V and V338F) identified. Mutations in brief no. 220. Online. Hum Mutat 1999:13:173.
14. Seydewitz $\mathrm{HH}$, Matern D. Molecular genetic analysis of 40 patients with glycogen storage disease type la: $100 \%$ mutation detection rate and 5 novel mutations. Hum Mutat 2000;15:115-116.

15. Stroppiano M, Regis S, DiRocco M, Caroli F, Gandullia P, Gatti R. Mutations in the glucose-6-phosphatase gene of 53 Italian patients with glycogen storage disease type la. J Inherit Metab Dis 1999;22:43-49.

16. Chou JY, Mansfield BC. Mutations in the glucose-6-phosphatase-alpha (G6PC) gene that cause type la glycogen storage disease. Hum Mutat 2008;29:921-930.

17. Veiga-da-Cunha M, Gerin I, Chen YT, de Barsy T, de Lonlay P, Dionisi-Vici C, et al. A gene on chromosome $11 \mathrm{q} 23$ coding for a putative glucose- 6-phosphate translocase is mutated in glycogen-storage disease types $\mathrm{lb}$ and Ic. Am J Hum Genet 1998;63:976-983.

18. Kishnani P, Koeberl D, Chen Y. Glycogen storage diseases. In: Valle D, Beaudet A, Vogelstein B, Kinzler K, Antonarakis S, Ballabio A (eds). Scriver's Online Metabolic \& Molecular Bases of Inherited Disease. McGraw-Hill: New York, 2009:71

19. Cassiman D, Libbrecht L, Verslype C, et al. An adult male patient with multiple adenomas and a hepatocellular carcinoma: mild glycogen storage disease type la. J Hepatol 2010;53:213-217.

20. Keller KM, Schütz M, Podskarbi T, Bindl L, Lentze MJ, Shin YS. A new mutation of the glucose-6-phosphatase gene in a 4-year-old girl with oligosymptomatic glycogen storage disease type 1a. J Pediatr 1998;132:360-361.

21. Lee P, Mather S, Owens C, Leonard J, Dicks-Mireaux C. Hepatic ultrasound findings in the glycogen storage diseases. Br J Radiol 1994;67:1062-1066.

22. Reddy SK, Austin SL, Spencer-Manzon M, et al. Liver transplantation for glycogen storage disease type la. J Hepatol 2009;51:483-490.

23. Reddy SK, Kishnani PS, Sullivan JA, et al. Resection of hepatocellular adenoma in patients with glycogen storage disease type la. J Hepatol 2007;47:658-663.

24. Franco LM, Krishnamurthy V, Bali D, et al. Hepatocellular carcinoma in glycogen storage disease type la: a case series. J Inherit Metab Dis 2005;28:153-162.

25. Kelly PM, Poon FW. Hepatic tumours in glycogen storage disease type 1 (von Gierke's disease). Clin Radio/ 2001;56:505-508.

26. Kudo M. Hepatocellular adenoma in type la glycogen storage disease. J Gastroentero/ 2001;36:65-66.

27. Czapek EE, Deykin D, Salzman EW. Platelet dysfunction in glycogen storage disease type I. Blood 1973:41:235-247.

28. Mühlhausen C, Schneppenheim R, Budde U, et al. Decreased plasma concentration of von Willebrand factor antigen (VWF:Ag) in patients with glycogen storage disease type la. J Inherit Metab Dis 2005;28:945-950.

29. Weinstein DA, Roy CN, Fleming MD, Loda MF, Wolfsdorf JI, Andrews NC. Inappropriate expression of hepcidin is associated with iron refractory anemia: implications for the anemia of chronic disease. Blood 2002;100:3776-3781.

30. Banugaria SG, Austin SL, Boney A, Weber TJ, Kishnani PS. Hypovitaminosis D in glycogen storage disease type I. Mol Genet Metab 2010;99:434-437.

31. Cabrera-Abreu J, Crabtree NJ, Elias E, Fraser W, Cramb R, Alger S. Bone mineral density and markers of bone turnover in patients with glycogen storage disease types I, III and IX. J Inherit Metab Dis 2004;27:1-9.

32. Rake JP, Visser G, Huismans D, et al. Bone mineral density in children, adolescents and adults with glycogen storage disease type la: a cross-sectional and longitudinal study. J Inherit Metab Dis 2003:26:371-384.

33. Rake JP, Visser G, Labrune P, Leonard JV, Ullrich K, Smit GP. Glycogen storage disease type I: diagnosis, management, clinical course and outcome. Results of the European Study on Glycogen Storage Disease Type I (ESGSD I). Eur J Pediatr 2002;161(suppl 1):S20-S34

34. Schwahn B, Rauch F, Wendel U, Schönau E. Low bone mass in glycogen storage disease type 1 is associated with reduced muscle force and poor metabolic control. J Pediatr 2002;141:350-356.

35. Wolfsdorf J. Bones benefit from better biochemical control in type 1 glycogen storage disease. J Pediatr 2002;141:308-310.

36. Minarich LA, Kirpich A, Fiske LM, Weinstein DA. Bone mineral density in glycogen storage disease type la and Ib. Genet Med 2012;14:737-741.

37. Chen YT, Scheinman JI, Park HK, Coleman RA, Roe CR. Amelioration of proximal renal tubular dysfunction in type I glycogen storage disease with dietary therapy. N Engl J Med 1990;323:590-593.

38. Reitsma-Bierens WC. Renal complications in glycogen storage disease type I. Eur J Pediatr 1993;152(suppl 1):S60-S62

39. Reitsma-Bierens WC, Smit GP, Troelstra JA. Renal function and kidney size in glycogen storage disease type I. Pediatr Nephrol 1992;6:236-238.

40. Weinstein DA, Somers MJ, Wolfsdorf JI. Decreased urinary citrate excretion in type 1a glycogen storage disease. J Pediatr 2001;138:378-382. 
41. Marega A, Fregonese $C$, Tulissi $P$, et al. Preemptive liver-kidney transplantation in von Gierke disease: a case report. Transplant Proc 2011;43:1196-1197.

42. Labrune P. Glycogen storage disease type I: indications for liver and/or kidney transplantation. Eur J Pediatr 2002;161(suppl 1):S53-S55.

43. Froissart R, Piraud M, Boudjemline AM, et al. Glucose-6-phosphatase deficiency. Orphanet J Rare Dis 2011;6:27.

44. Sechi A, Deroma L, Lapolla A, et al. Fertility and pregnancy in women affected by glycogen storage disease type I, results of a multicenter Italian study. J Inherit Metab Dis 2013;36:83-89.

45. Austin SL, El-Gharbawy AH, Kasturi VG, James A, Kishnani PS. Menorrhagia in patients with type I glycogen storage disease. Obstet Gynecol 2013;122: $1246-1254$.

46. Lee PJ, Patel A, Hindmarsh PC, Mowat AP, Leonard JV. The prevalence of polycystic ovaries in the hepatic glycogen storage diseases: its association with hyperinsulinism. Clin Endocrinol (Oxf) 1995;42:601-606.

47. Martens DH, Rake JP, Schwarz M, et al. Pregnancies in glycogen storage disease type la. Am J Obstet Gynecol 2008;198:646.e1-646.e7.

48. Ryan IP, Havel RJ, Laros RK Jr. Three consecutive pregnancies in a patient with glycogen storage disease type IA (von Gierke's disease). Am J Obstet Gynecol 1994;170:1687-90; discussion 1690.

49. Humbert M, Labrune P, Simonneau G. Severe pulmonary arterial hypertension in type 1 glycogen storage disease. Eur J Pediatr 2002;161(suppl 1): S93-S96.

50. Kishnani P, Bengur AR, Chen YT. Pulmonary hypertension in glycogen storage disease type I. J Inherit Metab Dis 1996;19:213-216.

51. Goulart JM, Yoo JY, Kirchoff-Torres KF, Delman BN, Tuhrim S. Ischemic stroke in an adult with glycogen storage disease type I. J Clin Neurosci 2010;17: 1467-1469.

52. Visser G, Rake JP, Labrune P, et al.; European Study on Glycogen Storage Disease Type I. Consensus guidelines for management of glycogen storage disease type $1 \mathrm{~b}$ - European Study on Glycogen Storage Disease Type 1. Eur J Pediatr 2002;161(suppl 1):S120-S123.

53. Binkiewicz A, Senior B. Decreased ketogenesis in von Gierke's disease (type I glycogenosis). J Pediatr 1973;83:973-978.

54. Fernandes J, Pikaar NA. Ketosis in hepatic glycogenosis. Arch Dis Child 1972:47:41-46.

55. Zakon SJ, Oyamada A, Rosenthal IH. Eruptive xanthoma and hyperlipemia in glycogen storage disease (von Gierke's disease). AMA Arch Derm Syphilol 1953;67:146-151.

56. Corby DG, Putnam CW, Greene HL. Impaired platelet function in glucose-6phosphatase deficiency. J Pediatr 1974;85:71-76.

57. Hutton RA, Macnab AJ, Rivers RP. Defect of platelet function associated with chronic hypoglycaemia. Arch Dis Child 1976;51:49-55.

58. Wolfsdorf JI, Laffel LM, Crigler JF Jr. Metabolic control and renal dysfunction in type I glycogen storage disease. J Inherit Metab Dis 1997;20:559-568.

59. Chen YT. Type I glycogen storage disease: kidney involvement, pathogenesis and its treatment. Pediatr Nephrol 1991;5:71-76.

60. Lee PJ, Dalton RN, Shah V, Hindmarsh PC, Leonard JV. Glomerular and tubular function in glycogen storage disease. Pediatr Nephrol 1995;9:705-710.

61. Restaino I, Kaplan BS, Stanley C, Baker L. Nephrolithiasis, hypocitraturia, and a distal renal tubular acidification defect in type $1 \mathrm{glycogen}$ storage disease. J Pediatr 1993;122:392-396.

62. Chen YT, Coleman RA, Scheinman JI, Kolbeck PC, Sidbury JB. Renal disease in type I glycogen storage disease. N Eng/ J Med 1988;318:7-11.

63. Weinstein DA, Wolfsdorf Jl. Effect of continuous glucose therapy with uncooked cornstarch on the long-term clinical course of type 1a glycogen storage disease. Eur J Pediatr 2002;161(suppl 1):S35-S39.

64. Visser G, Rake JP, Fernandes J, et al. Neutropenia, neutrophil dysfunction, and inflammatory bowel disease in glycogen storage disease type lb: results of the European Study on Glycogen Storage Disease type I. J Pediatr 2000;137: 187-191.

65. Melis D, Pivonello R, Parenti G, et al. Increased prevalence of thyroid autoimmunity and hypothyroidism in patients with glycogen storage disease type I. J Pediatr 2007;150:300-5, 305.e1.

66. Clayton PT. Diagnosis of inherited disorders of liver metabolism. J Inherit Metab Dis 2003;26:135-146.

67. Fischer KF, Lees JA, Newman JH. Hypoglycemia in hospitalized patients. Causes and outcomes. N Engl J Med 1986;315:1245-1250.

68. Wolf AD, Lavine JE. Hepatomegaly in neonates and children. Pediatr Rev 2000;21:303-310.

69. van den Berghe G. Disorders of gluconeogenesis. J Inherit Metab Dis 1996;19:470-477.
70. Baker L, Winegrad Al. Fasting hypoglycaemia and metabolic acidosis associated with deficiency of hepatic fructose-1,6-diphosphatase activity. Lancet 1970;2:13-16.

71. Morris AA, Deshphande S, Ward-Platt MP, et al. Impaired ketogenesis in fructose-1,6-bisphosphatase deficiency: a pitfall in the investigation of hypoglycaemia. J Inherit Metab Dis 1995;18:28-32.

72. Santer R, Schneppenheim R, Suter D, Schaub J, Steinmann B. Fanconi-Bickel syndrome-the original patient and his natural history, historical steps leading to the primary defect, and a review of the literature. Eur J Pediatr 1998;157: 783-797.

73. Santer R, Steinmann B, Schaub J. Fanconi-Bickel syndrome-a congenital defect of facilitative glucose transport. Curr Mol Med 2002;2:213-227.

74. Berry GT, Baker L, Kaplan FS, Witzleben CL. Diabetes-like renal glomerular disease in Fanconi-Bickel syndrome. Pediatr Nephrol 1995;9:287-291.

75. Berry GT, Baynes JW, Wells-Knecht KJ, Szwergold BS, Santer R. Elements of diabetic nephropathy in a patient with GLUT 2 deficiency. Mol Genet Metab 2005;86:473-477.

76. Charpentier C, Saudubray J. Clinical phenotypes: diagnosis/algorithms. In: Valle D, Beaudet A, Vogelstein B, Kinzler K, Antonarakis S, Ballabio A (eds). Scriver's Online Metabolic \& Molecular Bases of Inherited Disease. McGraw-Hill: New York, 2009:66.

77. McAdams AJ, Hug G, Bove KE. Glycogen storage disease, types I to X: criteria for morphologic diagnosis. Hum Pathol 1974;5:463-487.

78. Coleman RA, Winter HS, Wolf B, Chen YT. Glycogen debranching enzyme deficiency: long-term study of serum enzyme activities and clinical features. J Inherit Metab Dis 1992;15:869-881.

79. Veiga-da-Cunha M, Gerin I, Chen YT, Lee PJ, Leonard JV, Maire I, et al. The putative glucose-6-phosphate translocase gene is mutated in essentially all cases of glycogen storage disease type I non-a. Eur J Hum Genet 1999;7: 717-723.

80. Kure S, Rolland MO, Leisti J, et al. Prenatal diagnosis of non-ketotic hyperglycinaemia: enzymatic diagnosis in 28 families and DNA diagnosis detecting prevalent Finnish and Israeli-Arab mutations. Prenat Diagn 1999;19:717-720.

81. Kojima K, Kure S, Kamada F, et al. Genetic testing of glycogen storage disease type Ib in Japan: five novel G6PT1 mutations and a rapid detection method for a prevalent mutation W118R. Mol Genet Metab 2004;81:343-346.

82. Veiga-da-Cunha M, Gerin I, Van Schaftingen E. How many forms of glycogen storage disease type I? Eur J Pediatr 2000;7:717-723.

83. Janecke $A R$, Lindner $M$, Erdel $M$, et al. Mutation analysis in glycogen storage disease type 1 non-a. Hum Genet 2000;107:285-289.

84. Rake JP, Visser G, Labrune P, Leonard JV, Ullrich K, Smit GP; European Study on Glycogen Storage Disease Type I (ESGSD I). Guidelines for management of glycogen storage disease type I - European Study on Glycogen Storage Disease Type I (ESGSD I). Eur J Pediatr 2002;161 (suppl 1):S112-S119.

85. Bhattacharya K, Orton RC, Qi X, et al. A novel starch for the treatment of glycogen storage diseases. J Inherit Metab Dis 2007;30:350-357.

86. Correia CE, Bhattacharya K, Lee PJ, et al. Use of modified cornstarch therapy to extend fasting in glycogen storage disease types la and Ib. Am J Clin Nutr 2008;88:1272-1276.

87. Kishnani PS. Glycogen storage diseases. In: Ekvall SW, Ekvall VK (eds). Pediatric Nutrition in Chronic Diseases and Developmental Disorders: Prevention, Assessment, and Treatment, 2nd edn. Oxford University Press: New York, 2005: 350-355.

88. Wolfsdorf JI, Weinstein DA. Glycogen storage diseases. Rev Endocr Metab Disord 2003;4:95-102.

89. Goldberg T, Slonim AE. Nutrition therapy for hepatic glycogen storage diseases. J Am Diet Assoc 1993;93:1423-1430.

90. Fernandes J, van de Kamer JH. [Glucose, fructose and galactose in the diet of patients with glycogen accumulation disease type I]. Maandschr Kindergeneeskd 1964;32:29-34.

91. Fernandes $J$. The effect of disaccharides on the hyperlactacidaemia of glucose6-phosphatase-deficient children. Acta Paediatr Scand 1974;63:695-698.

92. Bier DM, Leake RD, Haymond MW, et al. Measurement of "true" glucose production rates in infancy and childhood with 6,6-dideuteroglucose. Diabetes 1977; 26:1016-1023.

93. Tsalikian E, Simmons P, Gerich JE, Howard C, Haymond MW. Glucose production and utilization in children with glycogen storage disease type I. Am J Physiol 1984;247(4 Pt 1):E513-E519.

94. Schwenk WF, Haymond MW. Optimal rate of enteral glucose administration in children with glycogen storage disease type I. N Engl J Med 1986;314: 682-685. 
95. Leonard JV, Dunger DB. Hypoglycaemia complicating feeding regimens for glycogen-storage disease. Lancet 1978;2:1203-1204.

96. Kishnani PS, Boney A, Chen YT. Nutritional deficiencies in a patient with glycogen storage disease type Ib. J Inherit Metab Dis 1999;22:795-801.

97. Bhattacharya K. Dietary dilemmas in the management of glycogen storage disease type I. J Inherit Metab Dis 2011;34:621-629.

98. Chen YT, Cornblath M, Sidbury JB. Cornstarch therapy in type I glycogenstorage disease. NEngl J Med 1984;310:171-175.

99. Franceschini R, Gianetta E, Pastorino A, et al. Crohn's-like colitis in glycogen storage disease $\mathrm{lb}$ : a case report. Hepatogastroenterology 1996;43:1461-1464.

100. Dieckgraefe BK, Korzenik JR, Husain A, Dieruf L. Association of glycogen storage disease $1 \mathrm{~b}$ and Crohn disease: results of a North American survey. Eur J Pediatr 2002;161(suppl 1):S88-S92.

101. Sidbury JB, Chen YT, Roe CR. The role of raw starches in the treatment of type I glycogenosis. Arch Intern Med 1986;146:370-373.

102. Saunders AC, Feldman HA, Correia CE, Weinstein DA. Clinical evaluation of a portable lactate meter in type I glycogen storage disease. J Inherit Metab Dis 2005;28:695-701.

103. White FJ, Jones SA. The use of continuous glucose monitoring in the practical management of glycogen storage disorders. J Inherit Metab Dis 2011;34: 631-642.

104. Storch E, Keeley M, Merlo L, Jacob M, Correia C, Weinstein D. Psychosocial functioning in youth with glycogen storage disease type I. J Pediatr Psychol 2008;33:728-738.

105. Farber M, Knuppel RA, Binkiewicz A, Kennison RD. Pregnancy and von Gierke's disease. Obstet Gynecol 1976;47:226-228.

106. Johnson MP, Compton A, Drugan A, Evans MI. Metabolic control of von Gierke disease (glycogen storage disease type la) in pregnancy: maintenance of euglycemia with cornstarch. Obstet Gynecol 1990;75(3 Pt 2):507-510.

107. Mairovitz V, Labrune P, Fernandez H, Audibert F, Frydman R. Contraception and pregnancy in women affected by glycogen storage diseases. Eur $J$ Pediatr 2002;161(suppl 1):S97-S101.

108. Dagli Al, Lee PJ, Correia CE, et al. Pregnancy in glycogen storage disease type Ib: gestational care and report of first successful deliveries. J Inherit Metab Dis 2010;33(suppl 3):S151-S157.

109. Green D. Effect of fish oil on platelet adhesiveness. Blood 1991;77:207-208.

110. Levy E, Thibault L, Turgeon J, et al. Beneficial effects of fish-oil supplements on lipids, lipoproteins, and lipoprotein lipase in patients with glycogen storage disease type I. Am J Clin Nutr 1993;57:922-929.

111. Nagasaka H, Hirano K, Ohtake A, et al. Improvements of hypertriglyceridemia and hyperlacticemia in Japanese children with glycogen storage disease type la by medium-chain triglyceride milk. Eur J Pediatr 2007;166:1009-1016.

112. Das AM, Lücke T, Meyer U, Hartmann H, Illsinger S. Glycogen storage disease type 1: impact of medium-chain triglycerides on metabolic control and growth. Ann Nutr Metab 2010;56:225-232.

113. Melis D, Della Casa R, Parini R, et al. Vitamin E supplementation improves neutropenia and reduces the frequency of infections in patients with glycogen storage disease type 1b. Eur J Pediatr 2009;168:1069-1074.

114. Talente GM, Coleman RA, Alter C, et al. Glycogen storage disease in adults. Ann Intern Med 1994;120:218-226.

115. Chen YT. Glycogen storage disease. In: Scriver CR, Beaudet AL, Sly WS, Valle D (eds). The Metabolic and Molecular Bases of Inherited Disease, 3rd edn, vol. 1. McGraw-Hill: New York, 2001:1521-1552.

116. Smit GP. The long-term outcome of patients with glycogen storage disease type la. Eur J Pediatr 1993;152(suppl 1):S52-S55.

117. Lee PJ. Glycogen storage disease type I: pathophysiology of liver adenomas. Eur J Pediatr 2002;161(suppl 1):S46-S49.

118. Wang DQ, Fiske LM, Carreras CT, Weinstein DA. Natural history of hepatocellular adenoma formation in glycogen storage disease type I. J Pediatr 2011;159:442-446.

119. Lee PJ, Celermajer DS, Robinson J, McCarthy SN, Betteridge DJ, Leonard JV. Hyperlipidaemia does not impair vascular endothelial function in glycogen storage disease type 1a. Atherosclerosis 1994;110:95-100.

120. Kishnani PS, Chuang TP, Bali D, et al. Chromosomal and genetic alterations in human hepatocellular adenomas associated with type la glycogen storage disease. Hum Mol Genet 2009; 18:4781-4790.

121. Chen, YJ, Chen, PJ, Lee, MC, Yeh, SH, Hsu, MT, Lin, CH. Chromosomal analysis of hepatic adenoma and focal nodular hyperplasia by comparative genomic hybridization. Genes Chromosomes Cancer 2002;35:138-143.
122. Faivre L, Houssin D, Valayer J, Brouard J, Hadchouel M, Bernard O. Long-term outcome of liver transplantation in patients with glycogen storage disease type la. J Inherit Metab Dis 1999;22:723-732.

123. Yoshikawa M, Fukui K, Kuriyama S, et al. Hepatic adenomas treated with percutaneous ethanol injection in a patient with glycogen storage disease type la. J Gastroentero/ 2001;36:52-61.

124. Malatack JJ, Finegold DN, Iwatsuki S, et al. Liver transplantation for type I glycogen storage disease. Lancet 1983;1:1073-1075.

125. Maheshwari A, Rankin R, Segev DL, Thuluvath PJ. Outcomes of liver transplantation for glycogen storage disease: a matched-control study and a review of literature. Clin Transplant 2012;26:432-436.

126. O'Leary JG, Lepe R, Davis GL. Indications for liver transplantation. Gastroenterology 2008;134:1764-1776.

127. Wiesner R, Edwards E, Freeman R, et al.; United Network for Organ Sharing Liver Disease Severity Score Committee. Model for end-stage liver disease (MELD) and allocation of donor livers. Gastroenterology 2003;124: 91-96.

128. Merion RM, Schaubel DE, Dykstra DM, Freeman RB, Port FK, Wolfe RA. The survival benefit of liver transplantation. Am J Transplant 2005;5:307-313.

129. Ojo AO, Held PJ, Port FK, et al. Chronic renal failure after transplantation of a nonrenal organ. N Engl J Med 2003;349:931-940.

130. Matern D, Starzl T, Arnaout W, Barnard J, Bynon J, Dhawan A, et al. Liver transplantation for glycogen storage disease type I, III, and IV. Eur J Pediatr 1999;159(suppl 2):543.

131. Lachaux A, Boillot O, Stamm D, et al. Orthotopic liver transplantation for glycogen storage disease type Ib-treatment with recombinant human granulocyte colony-stimulating factor. Transplant Proc 1994;26:265.

132. Karaki C, Kasahara M, Sakamoto S, et al. Glycemic management in living donor liver transplantation for patients with glycogen storage disease type $1 \mathrm{~b}$. Pediatr Transplant 2012;16:465-470.

133. Mundy HR, Lee PJ. Glycogenosis type I and diabetes mellitus: a common mechanism for renal dysfunction? Med Hypotheses 2002;59:110-114.

134. Urushihara $M$, Kagami S, Ito $M$, et al. Transforming growth factorbeta in renal disease with glycogen storage disease I. Pediatr Nephrol 2004; 19:676-678.

135. Martens DH, Rake JP, Navis G, Fidler V, van Dael CM, Smit GP. Renal function in glycogen storage disease type I, natural course, and renopreservative effects of ACE inhibition. Clin J Am Soc Nephro/ 2009;4:1741-1746.

136. Yiu WH, Mead PA, Jun HS, Mansfield BC, Chou JY. Oxidative stress mediates nephropathy in type la glycogen storage disease. Lab Invest 2010;90:620-629.

137. Baker L, Dahlem S, Goldfarb S, et al. Hyperfiltration and renal disease in glycogen storage disease, type I. Kidney Int 1989;35:1345-1350.

138. Schwartz GJ, Muñoz A, Schneider MF, et al. New equations to estimate GFR in children with CKD. J Am Soc Nephrol 2009;20:629-637.

139. Levey AS, Coresh J, Greene T, et al.; Chronic Kidney Disease Epidemiology Collaboration. Using standardized serum creatinine values in the modification of diet in renal disease study equation for estimating glomerular filtration rate. Ann Intern Med 2006;145:247-254.

140. Bank N. Mechanisms of diabetic hyperfiltration. Kidney Int 1991;40:792807.

141. Anderson S, Jung FF, Ingelfinger JR. Renal renin-angiotensin system in diabetes: functional, immunohistochemical, and molecular biological correlations. Am J Physiol 1993;265(4 Pt 2):F477-F486.

142. Melis D, Parenti G, Gatti R, et al. Efficacy of ACE-inhibitor therapy on renal disease in glycogen storage disease type 1: a multicentre retrospective study. Clin Endocrinol (Oxf) 2005;63:19-25.

143. MacKinnon M, Shurraw S, Akbari A, Knoll GA, Jaffey J, Clark HD. Combination therapy with an angiotensin receptor blocker and an ACE inhibitor in proteinuric renal disease: a systematic review of the efficacy and safety data. Am J Kidney Dis 2006;48:8-20.

144. Wang DQ, Carreras CT, Fiske LM, et al. Characterization and pathogenesis of anemia in glycogen storage disease type la and Ib. Genet Med 2012;14: 795-799.

145. Marti GE, Rick ME, Sidbury J, Gralnick HR. DDAVP infusion in five patients with type la glycogen storage disease and associated correction of prolonged bleeding times. Blood 1986;68:180-184.

146. Dellinger TM, Livingston HM, Holder R, Streckfus CF. Glycogen storage disease and von Willebrand's disease implications for dental treatment: dental management of a pediatric patient. Spec Care Dentist 1998;18:243-246. 
147. Visser G, Rake JP, Labrune P, et al. Granulocyte colony-stimulating factor in glycogen storage disease type $1 \mathrm{~b}$. Results of the European Study on Glycogen Storage Disease Type 1. Eur J Pediatr 2002;161(suppl 1):S83-S87.

148. Melis D, Fulceri R, Parenti G, et al. Genotype/phenotype correlation in glycogen storage disease type $1 \mathrm{~b}$ : a multicentre study and review of the literature. Eur J Pediatr 2005;164:501-508.

149. Chou JY, Jun HS, Mansfield BC. Neutropenia in type Ib glycogen storage disease. Curr Opin Hemato/ 2010;17:36-42.

150. Jun HS, Lee YM, Cheung YY, et al. Lack of glucose recycling between endoplasmic reticulum and cytoplasm underlies cellular dysfunction in glucose-6-phosphatase-beta-deficient neutrophils in a congenital neutropenia syndrome. Blood 2010;116:2783-2792.

151. Kilpatrick L, Garty BZ, Lundquist KF, et al. Impaired metabolic function and signaling defects in phagocytic cells in glycogen storage disease type 1b. J Clin Invest 1990;86:196-202.

152. Schroten $\mathrm{H}$, Wendel U, Burdach S, et al. Colony-stimulating factors for neutropenia in glycogen storage disease lb. Lancet 1991;337:736737.

153. Roe TF, Coates TD, Thomas DW, Miller JH, Gilsanz V. Brief report: treatment of chronic inflammatory bowel disease in glycogen storage disease type lb with colony-stimulating factors. N Eng/ J Med 1992;326:1666-1669.

154. Yamaguchi T, Ihara K, Matsumoto T, et al. Inflammatory bowel diseaselike colitis in glycogen storage disease type 1b. Inflamm Bowel Dis 2001;7: 128-132.

155. Alsultan A, Sokol RJ, Lovell MA, Thurman G, Ambruso DR. Long term G-CSFinduced remission of ulcerative colitis-like inflammatory bowel disease in a patient with glycogen storage disease $\mathrm{Ib}$ and evaluation of associated neutrophil function. Pediatr Blood Cancer 2010;55:1410-1413.

156. Ubels FL, Rake JP, Slaets JPJ, Smit GPA, Smit AJ. Is glycogen storage disease 1a associated with atherosclerosis? Eur J Pediatr 2002;161(suppl 1):S62-S64

157. Pizzo CJ. Type I glycogen storage disease with focal nodular hyperplasia of the liver and vasoconstrictive pulmonary hypertension. Pediatrics 1980;65: 341-343.

158. Furukawa N, Kinugasa A, Inoue F, Imashuku S, Takamatsu T, Sawada T. Type I glycogen storage disease with vasoconstrictive pulmonary hypertension. $J$ Inherit Metab Dis 1990;13:102-107.

159. Hamaoka K, Nakagawa M, Furukawa N, Sawada T. Pulmonary hypertension in type I glycogen storage disease. Pediatr Cardiol 1990;11:54-56.

160. Ohura T, Inoue CN, Abukawa D, et al. Progressive pulmonary hypertension: a fatal complication of type I glycogen storage disease. J Inherit Metab Dis 1995; 18:361-362.

161. Bolz D, Stocker F, Zimmermann A. Pulmonary vascular disease in a child with atrial septal defect of the secundum type and type I glycogen storage disease. Pediatr Cardiol 1996;17:265-267.

162. Ueno M, Murakami T, Takeda A, Kubota M. Efficacy of oral sildenafil in a beraprost-treated patient with severe pulmonary hypertension secondary to type I glycogen storage disease. Circ J 2009;73:1965-1968.

163. Milan A, Magnino C, Veglio F. Echocardiographic indexes for the noninvasive evaluation of pulmonary hemodynamics. J Am Soc Echocardiogr 2010;23:225-239; quiz 332.

164. Ben Salem C, Fathallah N, Hmouda H, Bouraoui K. Drug-induced hypoglycaemia: an update. Drug Saf 2011;34:21-45.

165. Khoza S, Barner JC. Glucose dysregulation associated with antidepressant agents: an analysis of 17 published case reports. Int J Clin Pharm 2011;33:484-492.

166. Zand DJ, Brown KM, Lichter-Konecki U, Campbell JK, Salehi V, Chamberlain JM. Effectiveness of a clinical pathway for the emergency treatment of patients with inborn errors of metabolism. Pediatrics 2008;122:1191-1195.

167. Lipper J, Weinstein DA, Taub PJ. Perioperative management of patients with glycogen storage disease type la. Plast Reconstr Surg 2008;122:42e-43e.

168. Huang IR, Jean WH, Lu CW, et al. Two different anesthetic managements of a patient with von Gierke's disease. Acta Anaesthesiol Taiwan 2006;:44:51-53.

169. Oshita A, Itamoto T, Amano H, Ohdan H, Tashiro H, Asahara T. Perioperative management of benign hepatic tumors in patients with glycogen storage disease type la. J Hepatobiliary Pancreat Surg 2008;15:200-203.

170. Giannitrapani L, Soresi M, La Spada E, Cervello M, D'Alessandro N, Montalto G. Sex hormones and risk of liver tumor. Ann NY Acad Sci 2006;1089:228-236.

171. Bahamondes L, Monteiro-Dantas C, Espejo-Arce X, et al. A prospective study of the forearm bone density of users of etonorgestrel- and levonorgestrelreleasing contraceptive implants. Hum Reprod 2006;21:466-470.
172. Mottet $C$, Juillerat $P$, Pittet $V$, et al. Pregnancy and breastfeeding in patients with Crohn's disease. Digestion 2007;76:149-160.

173. Terkivatan T, de Wilt JH, de Man RA, ljzermans JN. Management of hepatocellular adenoma during pregnancy. Liver 2000;20:186-187.

174. Abe T, Azuma H, Watanabe A, et al. A patient with cyclic neutropenia complicated by severe persistent neutropenia successfully delivered a healthy baby. Intern Med 2000;39:663-666.

175. Calhoun DA, Rosa C, Christensen RD. Transplacental passage of recombinant human granulocyte colony-stimulating factor in women with an imminent preterm delivery. Am J Obstet Gynecol 1996;174:1306-1311.

176. Dale DC, Cottle TE, Fier CJ, et al. Severe chronic neutropenia: treatment and follow-up of patients in the Severe Chronic Neutropenia International Registry. Am J Hematol 2003;72:82-93.

177. Fujiwaki R, Hata T, Hata K, Kitao M, Furuya H, Katoh Y. Effective treatment of drug-induced agranulocytosis using recombinant human granulocyte colony stimulating factor in pregnancy. Gynecol Obstet Invest 1995;40: 276-277.

178. Fung YL, Pitcher LA, Taylor K, Minchinton RM. Managing passively acquired autoimmune neonatal neutropenia: a case study. Transfus Med 2005;15: 151-155.

179. Kaufmann SJ, Sharif K, Sharma V, McVerry BA. Term delivery in a woman with severe congenital neutropenia, treated with growth colony stimulating factor. Hum Reprod 1998;13:498-499.

180. Sangalli MR, Peek M, McDonald A. Prophylactic granulocyte colony-stimulating factor treatment for acquired chronic severe neutropenia in pregnancy. Aust $N$ ZJ Obstet Gynaecol 2001;41:470-471.

181. Kim SY, Jun HS, Mead PA, Mansfield BC, Chou JY. Neutrophil stress and apoptosis underlie myeloid dysfunction in glycogen storage disease type $\mathrm{lb}$. Blood 2008;111:5704-5711.

182. Kim SY, Weinstein DA, Starost MF, Mansfield BC, Chou JY. Necrotic foci, elevated chemokines and infiltrating neutrophils in the liver of glycogen storage disease type la. J Hepatol 2008;48:479-485.

183. Koeberl DD, Pinto C, Sun B, et al. AAV vector-mediated reversal of hypoglycemia in canine and murine glycogen storage disease type la. Mol Ther 2008;16:665-672.

184. Yiu WH, Pan CJ, Ruef RA, et al. Angiotensin mediates renal fibrosis in the nephropathy of glycogen storage disease type la. Kidney Int 2008;73: 716-723.

185. Bandsma RH, Prinsen BH, van Der Velden Mde S, et al. Increased de novo lipogenesis and delayed conversion of large VLDL into intermediate density lipoprotein particles contribute to hyperlipidemia in glycogen storage disease type 1a. Pediatr Res 2008;63:702-707.

186. Lee KW, Lee JH, Shin SW, et al. Hepatocyte transplantation for glycogen storage disease type lb. Cell Transplant 2007;16:629-637.

187. Ribes-Koninckx C, Ibars EP, Calzado Agrasot MÁ, et al. Clinical outcome of hepatocyte transplantation in four pediatric patients with inherited metabolic diseases. Cell Transplant 2012;21:2267-2282.

188. Koeberl DD, Kishnani PS, Bali D, Chen YT. Emerging therapies for glycogen storage disease type I. Trends Endocrinol Metab 2009;20:252-258.

189. Yiu WH, Lee YM, Peng WT, et al. Complete normalization of hepatic G6PC deficiency in murine glycogen storage disease type la using gene therapy. Mol Ther 2010;18:1076-1084.

190. Grinshpun A, Condiotti R, Waddington SN, et al. Neonatal gene therapy of glycogen storage disease type la using a feline immunodeficiency virus-based vector. Mol Ther 2010;18:1592-1598.

191. Yiu WH, Pan CJ, Mead PA, Starost MF, Mansfield BC, Chou JY. Normoglycemia alone is insufficient to prevent long-term complications of hepatocellular adenoma in glycogen storage disease type Ib mice. J Hepatol 2009;51: 909-917.

192. Luo X, Hall G, Li S, et al. Hepatorenal correction in murine glycogen storage disease type I with a double-stranded adeno-associated virus vector. Mol Ther 2011;19:1961-1970.

193. Lee YM, Jun HS, Pan CJ, et al. Prevention of hepatocellular adenoma and correction of metabolic abnormalities in murine glycogen storage disease type la by gene therapy. Hepatology 2012;56:1719-1729.

194. Koeberl DD, Sun B, Bird A, Chen YT, Oka K, Chan L. Efficacy of helperdependent adenovirus vector-mediated gene therapy in murine glycogen storage disease type la. Mol Ther 2007;15:1253-1258.

195. Raju BI, Leyvi E, Seip R, et al. Enhanced gene expression of systemically administered plasmid DNA in the liver with therapeutic ultrasound and microbubbles. IEEE Trans Ultrason Ferroelectr Freq Control 2013;60: 88-96. 
196. Weinstein DA, Correia CE, Conlon T, et al. Adeno-associated virus-mediated correction of a canine model of glycogen storage disease type la. Hum Gene Ther 2010;21:903-910.

197. Demaster A, Luo X, Curtis S, et al. Long-term efficacy following readministration of an adeno-associated virus vector in dogs with glycogen storage disease type la. Hum Gene Ther 2012;23:407-418.

198. Brooks ED, Little $D$, Arumugam R, et al. Pathogenesis of growth failure and partial reversal with gene therapy in murine and canine glycogen storage disease type la. Mol Genet Metab 2013;109:161-170.

199. Mutel E, Abdul-Wahed A, Ramamonjisoa N, et al. Targeted deletion of liver glucose- 6 phosphatase mimics glycogen storage disease type 1a including development of multiple adenomas. J Hepatol 2011;54: 529-537.

200. Manno CS, Pierce GF, Arruda VR, et al. Successful transduction of liver in hemophilia by AAV-Factor IX and limitations imposed by the host immune response. Nat Med 2006;12:342-347.
201. Nathwani AC, Tuddenham EG, Rangarajan S, et al. Adenovirus-associated virus vector-mediated gene transfer in hemophilia B. N Engl J Med 2011;365:23572365.

202. Hwu WL, Chuang SC, Tsai LP, Chang MH, Chuang SM, Wang TR. Glucose 6-phosphatase gene G327A mutation is common in Chinese patients with glycogen storage disease type la. Hum Mol Genet 1995;4:1095-1096.

203. Nakamura T, Ozawa T, Kawasaki T, et al. Case report: hepatocellular carcinoma in type 1a glycogen storage disease with identification of a glucose-6-phosphatase gene mutation in one family. J Gastroenterol Hepatol 1999;14:553-558.

204. Santer R, Rischewski J, Block G, et al. Molecular analysis in glycogen storage disease 1 non-A: DHPLC detection of the highly prevalent exon 8 mutations of the G6PT1 gene in German patients. Hum Mutat 2000;16:177.

205. Annabi B, Hiraiwa H, Mansfield BC, et al. The gene for glycogen-storage disease type 1b maps to chromosome 11q23. Am J Hum Genet 1998;62: 400-405. 